\title{
The Pavement as a Waveguide: Modeling, System Identification, and Parameter Estimation
}

\author{
Roland Hostettler, Magnus Lundberg Nordenvaad, Wolfgang Birk
}

This is a post-print of a paper published in IEEE Transactions on Instrumentation and Measurement. When citing this work, you must always cite the original article:

R. Hostettler, M. Lundberg Nordenvaad, and W. Birk, "The pavement as a waveguide: Modeling, system identification, and parameter estimation," Instrumentation and Measurement, IEEE Transactions on, vol. 63, no. 8, pp. 2052-2063, 2014

\section{DOI:}

10.1109/TIM.2014.2304354

\section{Copyright:}

(C) 2014 IEEE. Personal use of this material is permitted. Permission from IEEE must be obtained for all other uses, in any current or future media, including reprinting/republishing this material for advertising or promotional purposes, creating new collective works, for resale or redistribution to servers or lists, or reuse of any copyrighted component of this work in other works. 


\title{
The Pavement as a Waveguide: Modeling, System Identification, and Parameter Estimation
}

\author{
Roland Hostettler, Student Member, IEEE, Magnus Lundberg Nordenvaad, Member, IEEE, \\ Wolfgang Birk, Member, IEEE
}

\begin{abstract}
This article presents modeling of wave propagation in pavements from a system identification point of view. First, a model based on the physical structure is derived. Second, experiment design and evaluation is discussed and maximum likelihood estimators for estimating the model parameters are introduced, assuming an error-in-variables setting. Finally, the proposed methods are applied to measurement data from two experiments under varying environmental conditions. It is found that the proposed methods can be used to estimate the dispersion curves of the considered waveguide and the results can be used for further analysis.
\end{abstract}

Index Terms-Modeling, system identification, parameter estimation, maximum likelihood estimation, surface waves

\section{INTRODUCTION}

Wave propagation in pavements is of interest in many different applications. For example, in non-destructive testing (NDT), the dynamic response of a pavement is measured and then used to assess the mechanical properties of the pavement. This is useful for example for wear prediction and maintenance [2], [3]. Other application areas that make use of the pavement's response to dynamic loads include weigh-in-motion (WIM) systems [4], or, more recently, traffic monitoring systems [5], [6]. In the latter, the seismic activity caused by vehicles passing a sensor is processed in order to estimate traffic and vehicle parameters of interest.

In both applications, a thorough understanding and modeling of the underlying phenomena as well as experimental design and evaluation are of great importance. Thus, understanding wave propagation in pavements becomes an apparent measurement and system identification problem. Once a theoretical model is derived, it is essential to determine the model parameters from experiments in order to be able to draw the desired conclusions. Furthermore, it is also important to design the excitation in a way so that the measurements make it possible to actually estimate the required parameters.

Many different approaches for both conducting experiments and gathering measurement data in pavements as well as evaluation have been developed during the past decades. The experimental setup most often uses either a static method such as a falling weight deflector (FWD) [7], [8] or a dynamic method using surface waves with different configurations on the source

This work has been supported by the Swedish Governmental Agency for Innovation Systems (VINNOVA), and GEVEKO AB.

R. Hostettler and W. Birk are with the Division of Systems and Interaction, Luleå University of Technology, Sweden.

M. Lundberg Nordenvaad is with the Division of Systems and Control, Uppsala University, Sweden. or the receiver side [9]-[11]. Evaluation of the experiments has been tackled using many different approaches [12], [13], ranging from purely analytical methods [14], [15], over genetic algorithms [16], [17] and neural networks [18] to a combination of the latter two [19].

Even though there is a broad amount of literature treating the problem at hand, most efforts neglect inherent factors such as measurement noise and disturbances. It is thus the goal of this work to approach the problem using a system identification framework that takes these problems into account. The contribution are estimation methods based on simplistic experiments. The results obtained from the proposed methods could then be used for further interpretation or in other inversion methods as mentioned above.

The remainder of this article is organized as follows. First, a short introduction to wave propagation in a plate as a waveguide is given in Section II, followed by the derivation of the system identification and parameter estimation methods in Section III. The proposed estimators are then simulated in Section IV and the theory is applied to experiments and evaluated in Section V. Some concluding remarks follow in Section VI.

\section{WAVEGUIDE MODEL}

Wave propagation problems have been studied for a long time and in many different contexts. The level of complexity depends on the waveguide and can vary from trivial to extremely difficult. The problem considered here is wave propagation in roads. Thus, the waveguide is a multilayered structure consisting of an asphalt concrete top layer and one or more subgrade layers as illustrated in Fig. 1. Note that the top layer can be a layered structure in itself due to periodic resurfacing where a thin layer is fined down and replaced with new asphalt concrete to improve riding comfort.

The simplest way of looking at this problem is by neglecting the subgrade layers and simply assuming that the waveguide consists of a free plate. In practice, this means assuming traction free boundaries at the top-plates interface $z=-h / 2$ (Fig. 1). Clearly, this is a quite strong simplification but it will be clear from the derivation in the following sections that this is a reasonable assumption. The actual boundary conditions would dictate matching particle displacements at the layer interfaces.

Research on waves in free plates has been plentiful ever since the first paper by Lamb in 1917 [20]. Not surprisingly, the predominant waves in plates are now commonly called 


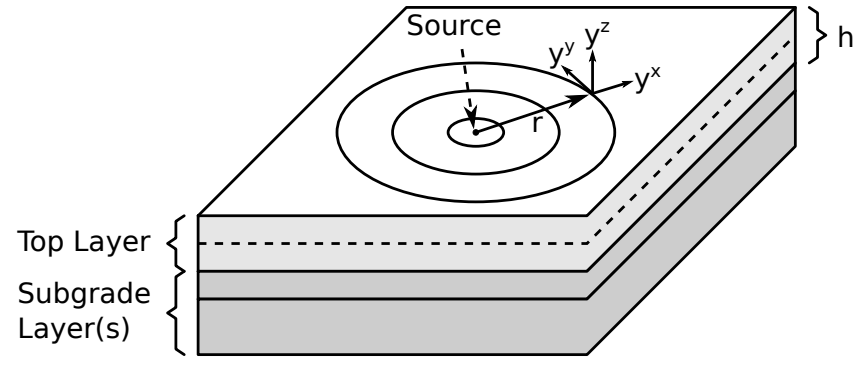

Fig. 1: Illustration of a cross-section of a road with an asphalt concrete top plate supported by one or more subgrade layers. The dashed line indicates the midplane of the top plate which is chosen as $z=0$. Circular wave propagation from a source is assumed and the particle motion at some point $r$ from the source is illustrated.

Lamb waves. Lamb waves describe longitudinal waves with their particle motion in-plane in the direction of propagation and vertical shear waves that have the particle motion out of plane, perpendicular to the direction of propagation. Apart from these two wave types, shear horizontal (SH) waves have also been observed. SH waves are characterized by a particle motion in-plane, perpendicular to the direction of propagation. Both types of waves have received a great deal of attention in the research community and hence, a complete treatment is avoided here. Instead, only the essential steps relevant to this application will be given in the following two sections. For an in-depth study of the mechanical problem and intricacies, the reader is referred to, for example, [20]-[23].

\section{A. Lamb Waves}

The first and predominant wave type mentioned above are the so-called Lamb waves which have their particle motion in the longitudinal $(x)$ and vertical shear $(z)$ directions. Lamb waves can be seen as the product of waves reflected at the top and bottom plate boundaries, resulting in infinite many different modes. Most commonly, the problem is approached using Helmholz decomposition. Solving the homogeneous equation system of the two potentials $\Phi$ and $\Psi$ given by

$$
\begin{aligned}
& \nabla^{2} \Phi-\left(\frac{\omega^{2}}{c_{L}^{2}}+i \omega \bar{\eta}_{L}\right) \Phi=0 \\
& \nabla^{2} \Psi-\left(\frac{\omega^{2}}{c_{T}^{2}}+i \omega \bar{\eta}_{T}\right) \Psi=0
\end{aligned}
$$

yields the well known solutions that exhibit modes that are symmetric and antisymmetric in their amplitude with respect to the midplane of the plate. In (1), $i$ is the imaginary unit, $\omega$ is the circular frequency, $c_{L}$ and $c_{T}$ are the longitudinal and transversal bulk wave velocities, and $\bar{\eta}_{L}$ and $\bar{\eta}_{T}$ the longitudinal and transversal viscous damping, respectively. Furthermore, by imposing the boundary conditions (traction free surfaces at $z= \pm h / 2$ ), the dispersion relation [23, pp. 106ff] is obtained. Unfortunately, the latter can only be solved numerically and hence the problem becomes difficult to solve.

However, assuming a point source which results in cylindrical wave spread, the particle displacement for any mode at the surface $z= \pm h / 2$ can be expressed by

$$
\begin{aligned}
& Y^{x}(r, \omega)=A^{x}(\omega) \mathcal{H}_{0}^{(1)}(-k r) U(\omega) \\
& Y^{z}(r, \omega)=A^{z}(\omega) \mathcal{H}_{0}^{(1)}(-k r) U(\omega)
\end{aligned}
$$

where $Y^{x}$ and $Y^{z}$ are the particle displacement in the longitudinal $(x)$ and vertical $(z)$ directions, respectively. $A^{x}$ and $A^{z}$ are the (complex) amplitudes in the respective direction, $r$ is the distance from the source to the point of measurement, $\mathcal{H}_{0}^{(1)}(x)$ is the Hankel function of the first kind of order 0 , $U(\omega)$ is the source spectrum, and

$$
k=k_{r}-i k_{i}=\frac{\omega}{c_{p}(\omega)}-i \eta(\omega)
$$

is the complex wavenumber where $c_{p}$ is the phase velocity and $\eta$ the attenuation coefficient. (2) can be rewritten as

$$
\begin{aligned}
{\left[\begin{array}{l}
Y^{x}(r, \omega) \\
Y^{z}(r, \omega)
\end{array}\right] } & =\left[\begin{array}{l}
A^{x}(\omega) \\
A^{z}(\omega)
\end{array}\right] \mathcal{H}_{0}^{(1)}(-k r) U(\omega) \\
& =\left[\begin{array}{l}
G^{x}(r, \omega) \\
G^{z}(r, \omega)
\end{array}\right] U(\omega)
\end{aligned}
$$

with

$$
\begin{aligned}
& G^{x}(r, \omega)=A^{x}(\omega) \mathcal{H}_{0}^{(1)}(-k r) \\
& G^{z}(r, \omega)=A^{z}(\omega) \mathcal{H}_{0}^{(1)}(-k r)
\end{aligned}
$$

\section{B. Shear Horizontal Waves}

The second kind of waves are shear horizontal (SH) waves. As opposed to the previous waves, the particle motion of SH waves is in-plane, but perpendicular to the direction of propagation. The assumptions for horizontal shear are simpler than for Lamb waves and result in the classical wave equation (spectral form) [23]

$$
\nabla^{2} Y^{y}-\left(\frac{\omega^{2}}{c_{T}^{2}}+i \omega \bar{\eta}_{T}\right) Y^{y}=0
$$

for the particle displacement $Y^{y}$ in y-direction. Even $\mathrm{SH}$ waves exhibit symmetric and antisymmetric modes with respect to the midplane $z=0$ of the plate. However, due to the simpler form of the dispersion relation (see [23, pp. 243ff]), the antisymmetric modes disappear at the surfaces $z= \pm h / 2$ and the solution to (5) reduces to

$$
Y^{y}(r, \omega)=A^{y}(\omega) \mathcal{H}_{0}^{(1)}\left(-k^{y} r\right) U(\omega)
$$

where

$$
k^{y}=k_{r}^{y}-i k_{i}^{y}=\frac{\omega}{c_{p}^{y}(\omega)}-i \eta^{y}(\omega)
$$

and $A^{y}$ is the complex amplitude, $c_{p}^{y}$ the phase velocity, and $\eta^{y}$ the attenuation coefficient, all in $y$-direction.

(6) can again be rewritten as

$$
Y^{y}(r, \omega)=G^{y}(r, \omega) U(\omega)
$$

with

$$
G^{y}(r, \omega)=A^{y}(\omega) \mathcal{H}_{0}^{(1)}\left(-k^{y} r\right)
$$

which is of the same form as (4). 


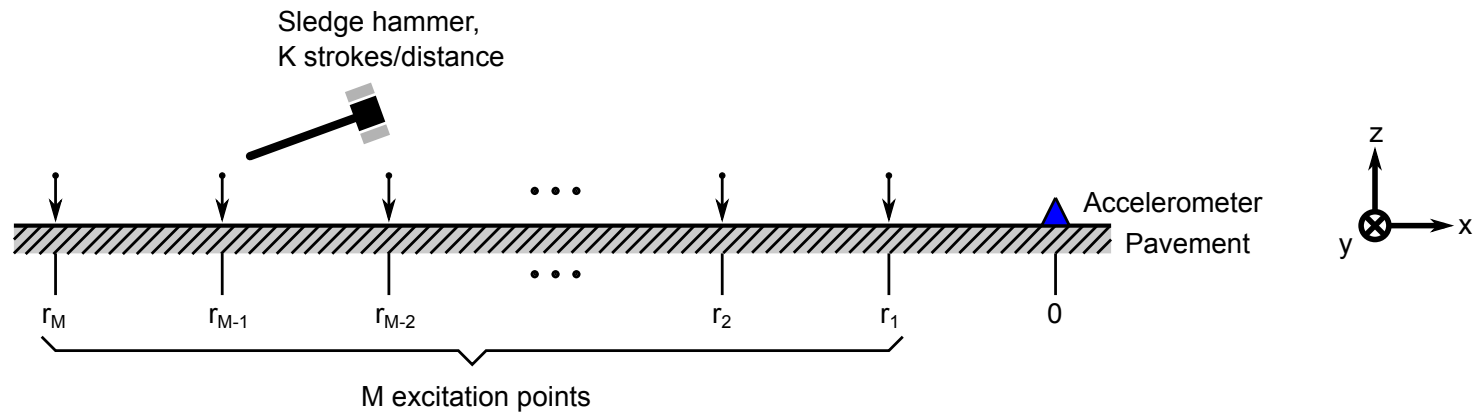

Fig. 2: Measurement setup for the pulse response measurements.

\section{SySTEM IDENTIFICATION}

\section{A. Experiment Design}

System identification for linear systems is a well-established domain and the literature on experiment design and evaluation is plentiful, see, for example [24] or [25] for thorough introductions to the field. An important aspect when designing system identification experiments is how to excite the system. Ideally, it should be excited in a broad frequency range so that all the important dynamics of the systems can be captured. To this end, random noise or random phase multisines are very well suited as excitation signals. Carefully designed, the latter type of signal is also an excellent choice for detecting possible non-linearities in the system [26]. However, it can sometimes prove difficult to use such an excitation since it might be impractical or impossible to apply this kind of excitation to a system due to different limiting factors and the practitioner has to resort to something else.

On the other hand, research in experimental pavement testing has been ongoing for the last 60 years or so and a variety of different testing methodologies have been developed. Some of the most popular methods include the spectral analysis of surface waves (SASW) [9], [27], multichannel analysis of surface waves (MASW) [10], or, more lately, the multichannel simulation with one receiver (MSOR) [28] methods. The setup used here is essentially a combination of the latter two which was also discussed in [11]. It is fairly simple and illustrated in Fig. 2. A sledge hammer is used to excite the structure at $M$ predefined distances $r_{m}(m=1, \ldots, M)$ from the sensor. At each distance, the experiment is repeated $K$ times and the response is measured using a triaxial accelerometer. The measured signals are the hammer force and the acceleration in the $x, y$, and $z$ directions. Even though the experiment setup is similar to the setup used in [11], the measurements are analyzed in a different way due to the different purpose.

Some of the biggest advantages of this setup are its simplicity. The fact that it can be built up easily means that experiments can be conducted rapidly and that it is nondestructive. An apparent and possibly major drawback is that it is hard to control the excitation bandwidth and energy accurately. A crude way to influence this is to use different contact pads which dampen the hammer upon impact.

Note that once the experiment is set up, the time to conduct the measurements is given by $K \cdot M$. For a fixed experiment time, this results in a trade-off between spatial information

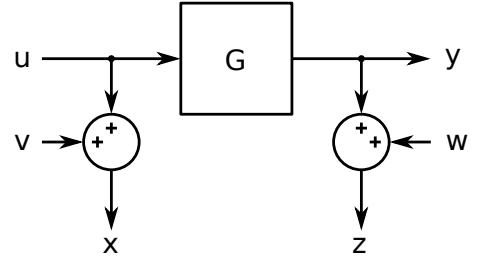

Fig. 3: Structure of the error-in-variables problem with the unknown system $G$, the true input $u$, the true output $y$, the measurement signals $x$ and $z$, and the measurement noises $v$ and $w$.

which contributes to the accuracy of the parameters of interest and averaging of the individual transfer functions as it will be shown in the following sections.

Finally, both, the input generated by the hammer as well as the output signals are measured and hence, they will both be disturbed by measurement noise. This has to be taken into account when analyzing the measurement data and estimating the frequency response function (FRF). One approach to do so is to use the error-in-variables framework as illustrated in Fig. 3.

\section{B. Non-Parametric Frequency Response Function}

Taking the above experimental setup and considerations into account, the problem can now be formalized. Starting from the fact that we are using an error-in-variables setting, the signal for the $k^{\text {th }}$ measurement at the $m^{\text {th }}$ distance is given by

$$
\begin{aligned}
x_{k}\left(r_{m}, n\right) & =u_{k}\left(r_{m}, n\right)+v(n) \\
z_{k}^{\alpha}\left(r_{m}, n\right) & =y_{k}^{\alpha}\left(r_{m}, n\right)+w(n) \\
& =g^{\alpha}\left(r_{m}, n\right) * u_{k}\left(r_{m}, n\right)+w(n)
\end{aligned}
$$

where $u_{k}\left(r_{m}, n\right)$ is the true excitation, $y_{k}^{\alpha}\left(r_{m}, n\right)$ the true output signal, and $x_{k}\left(r_{m}, n\right)$ and $z_{k}^{\alpha}\left(r_{m}, n\right)$ are the measured input and output signals, respectively. $g^{\alpha}\left(r_{m}, n\right)$ is the impulse response of the system and $*$ denotes the convolution. The superscript $\alpha$ indicates the component of the measurement vector and is one of $\{x, y, z\}$. Finally, the imperfections of the measurement equipment, thermal noise, etc. are modeled as white Gaussian noise, that is, $v(n) \sim \mathcal{N}\left(0, \sigma_{v}^{2}\right)$ for the input and $w(n) \sim \mathcal{N}\left(0, \sigma_{w}^{2}\right)$ for the output.

Since the measured input and output signals are both pulses, no windowing has to be applied to reduce leakage if it is 
ensured that the signals are measured from rest to rest. Hence, taking the unitary discrete Fourier transform (DFT) of (9) yields

$$
\begin{aligned}
X_{k}\left(r_{m}, \omega_{l}\right) & =U_{k}\left(r_{m}, \omega_{l}\right)+V\left(\omega_{l}\right) \\
Z_{k}^{\alpha}\left(r_{m}, \omega_{l}\right) & =G^{\alpha}\left(r_{m}, \omega_{l}\right) U_{k}\left(r_{m}, \omega_{l}\right)+W\left(\omega_{l}\right)
\end{aligned}
$$

in the Fourier domain. The noise components $V\left(\omega_{l}\right)$ and $W\left(\omega_{l}\right)$ are complex Gaussian distributed of the form $V\left(\omega_{l}\right) \sim$ $\mathcal{C N}\left(0, \sigma_{v}^{2}\right)$ and $W\left(\omega_{l}\right) \sim \mathcal{C N}\left(0, \sigma_{w}^{2}\right)$ for $l=1, \ldots, N / 2-1$, respectively [29]. The frequency of the $l^{\text {th }}$ bin is given by

$$
\omega_{l}=\frac{2 \pi l f_{s}}{N}
$$

where $N$ is the number of samples and $f_{s}$ is the sampling frequency. Note that the dependencies on $r_{m}$ and $\omega_{l}$ will be dropped for the sake of simplicity where not explicitly necessary from now on.

The $K$ measured independent input-output pairs for the distance $r_{m}$ yields $2 K$ measurement values that can be used to estimate the $K+1$ unknowns $U_{1}, \ldots, U_{K}$, and $G^{\alpha}$ for each $\omega_{l}$ of interest. It is straight forward that the joint probability density function (PDF) for $\mathbf{X}=\left[\begin{array}{lll}X_{1} & \ldots & X_{K}\end{array}\right]^{T}$ and $\mathbf{Z}^{\alpha}=\left[\begin{array}{lll}Z_{1}^{\alpha} & \ldots & Z_{K}^{\alpha}\end{array}\right]^{T}$ is

$$
\begin{aligned}
& p\left(\mathbf{X}, \mathbf{Z}^{\alpha} ; \mathbf{U}, G^{\alpha}\right) \\
& \quad=\mathcal{C N}\left(\left[\begin{array}{c}
\mathbf{X} \\
\mathbf{Z}^{\alpha}
\end{array}\right] ;\left[\begin{array}{c}
\mathbf{U} \\
G^{\alpha} \mathbf{U}
\end{array}\right],\left[\begin{array}{cc}
\mathbf{C}_{v v} & \mathbf{0} \\
\mathbf{0} & \mathbf{C}_{w w}
\end{array}\right]\right)
\end{aligned}
$$

where $\mathbf{U}=\left[\begin{array}{lll}U_{1} & \ldots & U_{K}\end{array}\right]^{T}$ and the multivariate complex Gaussian PDF $\mathcal{C N}(\mathbf{x} ; \boldsymbol{\mu}, \mathbf{C})$ is as defined in [29],

$$
\begin{aligned}
\mathbf{C}_{v v} & =\sigma_{v}^{2} \mathbf{I}_{K}, \\
\mathbf{C}_{w w} & =\sigma_{w}^{2} \mathbf{I}_{K},
\end{aligned}
$$

and $\mathbf{I}_{K}$ is the $K \times K$ identity matrix.

From (11), the maximum likelihood estimator (MLE) for $G^{\alpha}$ is given by (see Appendix)

$$
\hat{G}^{\alpha}=\frac{-b \pm \sqrt{b^{2}-4 a c}}{2 a}
$$

with

$$
\begin{aligned}
a & =\frac{1}{\sigma_{w}^{2}}\left(\mathbf{Z}^{\alpha}\right)^{H} \mathbf{X} \\
b & =\frac{1}{\sigma_{v}^{2}} \mathbf{X}^{H} \mathbf{X}-\frac{1}{\sigma_{w}^{2}}\left(\mathbf{Z}^{\alpha}\right)^{H} \mathbf{Z}^{\alpha} \\
c & =-\frac{1}{\sigma_{v}^{2}} \mathbf{X}^{H} \mathbf{Z}^{\alpha}
\end{aligned}
$$

and the superscript $H$ denotes the conjugate transpose.

Given the estimate $\hat{G}^{\alpha}$ from (12), the MLE for $\mathbf{U}$ is then [29]

$$
\hat{\mathbf{U}}=\left(\mathbf{H}^{H}\left(\hat{G}^{\alpha}\right) \mathbf{C}_{\mathcal{X}}^{-1} \mathbf{H}\left(\hat{G}^{\alpha}\right)\right)^{-1} \mathbf{H}^{H}\left(\hat{G}^{\alpha}\right) \mathbf{C}_{\mathcal{X}}^{-1} \mathcal{X}
$$

where

$$
\mathbf{H}\left(G^{\alpha}\right)=\left[\begin{array}{c}
\mathbf{I}_{K} \\
G^{\alpha} \mathbf{I}_{K}
\end{array}\right]
$$

and $\mathcal{X}$ and $\mathbf{C}_{\mathcal{X}}$ as defined in (25) in the Appendix.
Finally, the asymptotic covariance matrix of (12)-(13) is given by the inverse of the Fisher information matrix ((33) in the Appendix), that is,

$$
\begin{aligned}
\lim _{K \rightarrow \infty} \operatorname{cov}\left\{\hat{\mathbf{U}}, \hat{G}^{\alpha}\right\}=\mathcal{I}^{-1}\left(\mathbf{U}, G^{\alpha}\right) \\
=\left[\begin{array}{cc}
\mathbf{H}^{H} \mathbf{C}_{\mathcal{X}}^{-1} \mathbf{H} & \mathbf{H}^{H} \mathbf{C}_{\mathcal{X}}^{-1} \mathbf{H}^{\prime} \mathbf{U} \\
\mathbf{U}^{H} \mathbf{H}^{\prime H} \mathbf{C}_{\mathcal{X}}^{-1} \mathbf{H} & \mathbf{U}^{H} \mathbf{H}^{\prime H} \mathbf{C}_{\mathcal{X}}^{-1} \mathbf{H}^{\prime} \mathbf{U}
\end{array}\right]^{-1}
\end{aligned}
$$

which can be estimated by replacing the unknowns $G^{\alpha}$ and $\mathbf{U}$ with their estimates (12) and (13), respectively. Note that in practice, convergence is often attained for reasonable $K \ll$ $\infty$ [29] and the simulations in Section IV indicate that this is the case for $K \approx 10$.

Calculating (12) and (14) for every frequency bin $\omega_{l}$ and distance $r_{m}$ will finally yield an estimate of the complete nonparametric FRF $G^{\alpha}\left(r_{m}, \omega_{l}\right)$ and its variance.

\section{Semi-Parametric FRF Model}

Using the FRF estimates from the previous section and the analytical model for wave propagation introduced in Section II, the parameters of the latter can be estimated. This twostep procedure is similar to the method of moments estimation scheme [29] but differs in the way that instead of a moment matching, a new estimator is derived based on the distribution of the previously estimated parameters.

1) Lamb Waves: The analytical model for the transfer function for Lamb waves is given by (4) where $\mathbf{A}=\left[\begin{array}{ll}A^{x} & A^{z}\end{array}\right]^{T}$, and $k$ are unknown, complex, and frequency dependent parameters. From (12)-(14) we also know that asymptotically, $\hat{G}^{\alpha}\left(r_{m}, \omega_{l}\right) \sim \mathcal{C N}\left(G^{\alpha}\left(r_{m}, \omega_{l}\right),\left(\sigma_{m}^{\alpha}\right)^{2}\right)$ [29]. Letting

$$
\hat{\mathbf{G}}=\left[\begin{array}{c}
\hat{G}^{x}\left(r_{1}, \omega_{l}\right) \\
\vdots \\
\hat{G}^{x}\left(r_{M}, \omega_{l}\right) \\
\hat{G}^{z}\left(r_{1}, \omega_{l}\right) \\
\vdots \\
\hat{G}^{z}\left(r_{M}, \omega_{l}\right)
\end{array}\right]
$$

the joint PDF is modeled as

$$
p(\hat{\mathbf{G}} ; \mathbf{A}, k)=\mathcal{C N}\left(\hat{\mathbf{G}} ; \boldsymbol{\mu}_{\hat{\mathbf{G}}}(\mathbf{A}, k), \mathbf{C}_{\hat{\mathbf{G}}}\right)
$$


where

$$
\begin{aligned}
& \boldsymbol{\mu}_{\hat{\mathrm{G}}}(\mathbf{A}, k)= {\left[\begin{array}{c}
A^{x} \mathcal{H}_{0}^{(1)}\left(-k r_{1}\right) \\
\vdots \\
A^{x} \mathcal{H}_{0}^{(1)}\left(-k r_{M}\right) \\
A^{z} \mathcal{H}_{0}^{(1)}\left(-k r_{1}\right) \\
\vdots \\
A^{z} \mathcal{H}_{0}^{(1)}\left(-k r_{M}\right)
\end{array}\right] } \\
&= {\left[\begin{array}{cc}
\mathcal{H}_{0}^{(1)}\left(-k r_{1}\right) & 0 \\
\vdots & \vdots \\
\mathcal{H}_{0}^{(1)}\left(-k r_{M}\right) & 0 \\
0 & \mathcal{H}_{0}^{(1)}\left(-k r_{1}\right) \\
\vdots & \vdots \\
0 & \mathcal{H}_{0}^{(1)}\left(-k r_{M}\right)
\end{array}\right]\left[\begin{array}{l}
A^{x} \\
A^{z}
\end{array}\right] } \\
&= \mathbf{F}(k) \mathbf{A} \\
& \mathbf{C}_{\hat{\mathbf{G}}}=\left[\begin{array}{cc}
\mathbf{C}_{\hat{\mathbf{G}}}^{x} & \mathbf{0} \\
\mathbf{0} & \mathbf{C}_{\hat{\mathbf{G}}}^{z}
\end{array}\right],
\end{aligned}
$$

and

$$
\mathbf{C}_{\hat{\mathbf{G}}}^{\alpha}=\left[\begin{array}{cccc}
\left(\sigma_{1}^{\alpha}\right)^{2} & 0 & \cdots & 0 \\
0 & \left(\sigma_{2}^{\alpha}\right)^{2} & & \vdots \\
\vdots & & \ddots & 0 \\
0 & \cdots & 0 & \left(\sigma_{M}^{\alpha}\right)^{2}
\end{array}\right]
$$

The maximum likelihood estimates for $\mathbf{A}$, and $k$ are the maximizers of the exponent of (15) given by

$$
-\left(\hat{\mathbf{G}}-\boldsymbol{\mu}_{\hat{\mathbf{G}}}(\mathbf{A}, k)\right)^{H} \mathbf{C}_{\hat{\mathbf{G}}}^{-1}\left(\hat{\mathbf{G}}-\boldsymbol{\mu}_{\hat{\mathbf{G}}}(\mathbf{A}, k)\right)
$$

which is a separable problem since $\boldsymbol{\mu}_{\hat{\mathrm{G}}}$ is linear in $\mathbf{A}$. Therefore, $\hat{k}$ is found by maximizing [29]

$$
\begin{aligned}
\hat{k} & =\underset{k}{\operatorname{argmax}} J(k) \\
& =\underset{k}{\operatorname{argmax}} \hat{\mathbf{G}}^{H} \mathbf{C}_{\hat{\mathbf{G}}}^{-1} \mathbf{F}\left(\mathbf{F}^{H} \mathbf{C}_{\hat{\mathbf{G}}}^{-1} \mathbf{F}\right)^{-1} \mathbf{F}^{H} \mathbf{C}_{\hat{\mathbf{G}}}^{-1} \hat{\mathbf{G}}
\end{aligned}
$$

where $\mathbf{F} \triangleq \mathbf{F}(k)$. Once $\hat{k}$ is found, $\hat{\mathbf{A}}$ is given by

$$
\hat{\mathbf{A}}=\left(\mathbf{F}^{H}(\hat{k}) \mathbf{C}_{\hat{\mathbf{G}}}^{-1} \mathbf{F}(\hat{k})\right)^{-1} \mathbf{F}^{H}(\hat{k}) \mathbf{C}_{\hat{\mathbf{G}}}^{-1} \hat{\mathbf{G}}
$$

The stationary points for $\hat{k}$ in (19) are obtained by setting the gradient $\partial J(k) / \partial k^{*}=0$. The gradient is given by [30]

$$
\begin{aligned}
\frac{\partial J(k)}{\partial k^{*}}= & \hat{\mathbf{G}}^{H} \mathbf{C}_{\hat{\mathbf{G}}}^{-1} \mathbf{F}\left(\left(\frac{\partial}{\partial k^{*}}\left(\mathbf{F}^{H} \mathbf{C}_{\hat{\mathbf{G}}}^{-1} \mathbf{F}\right)^{-1}\right) \mathbf{F}^{H}\right. \\
& \left.+\left(\mathbf{F}^{H} \mathbf{C}_{\hat{\mathbf{G}}}^{-1} \mathbf{F}\right)^{-1} \frac{\partial}{\partial k^{*}} \mathbf{F}^{H}\right) \mathbf{C}_{\hat{\mathbf{G}}}^{-1} \hat{\mathbf{G}}
\end{aligned}
$$

where it was made use of the fact that $\mathbf{F}$ only depends on $k$ and not on its complex conjugate $k^{*}$. Furthermore,

$$
\begin{aligned}
\frac{\partial}{\partial k^{*}}\left(\mathbf{F}^{H} \mathbf{C}_{\hat{\mathbf{G}}}^{-1} \mathbf{F}\right)^{-1}= & -\left(\mathbf{F}^{H} \mathbf{C}_{\hat{\mathbf{G}}}^{-1} \mathbf{F}\right)^{-1} \\
& \cdot\left(\frac{\partial}{\partial k^{*}} \mathbf{F}^{H} \mathbf{C}_{\hat{\mathbf{G}}}^{-1} \mathbf{F}\right)\left(\mathbf{F}^{H} \mathbf{C}_{\hat{\mathbf{G}}}^{-1} \mathbf{F}\right)^{-1} .
\end{aligned}
$$

and the derivative of $\mathbf{F}^{H}$ with respect to $k^{*}$ is

$$
\frac{\partial \mathbf{F}^{H}}{\partial k^{*}}=\left(\frac{\partial \mathbf{F}}{\partial k}\right)^{H}=\left[\begin{array}{cc}
r_{1} \mathcal{H}_{1}^{(1)}\left(-k r_{1}\right) & 0 \\
\vdots & \vdots \\
r_{M} \mathcal{H}_{1}^{(1)}\left(-k r_{M}\right) & 0 \\
0 & r_{1} \mathcal{H}_{1}^{(1)}\left(-k r_{1}\right) \\
\vdots & \vdots \\
0 & r_{M} \mathcal{H}_{1}^{(1)}\left(-k r_{1}\right)
\end{array}\right] .
$$

Unfortunately, (21) does not exhibit a closed form solution. Therefore, a numerical root searching algorithm such as the well-known Newton-Raphson algorithm has to be employed.

The Hessian as defined in [30] is given by

$$
\begin{aligned}
\frac{\partial^{2} J(k)}{\partial k^{*} \partial k}= & \hat{\mathbf{G}}^{H} \mathbf{C}_{\hat{\mathbf{G}}}^{-1}\left(\mathbf{F}^{\prime}\left(\frac{\partial}{\partial k^{*}}\left(\mathbf{F}^{H} \mathbf{C}_{\hat{\mathbf{G}}}^{-1} \mathbf{F}\right)^{-1}\right) \mathbf{F}^{H}\right. \\
& +\mathbf{F}^{\prime}\left(\mathbf{F}^{H} \mathbf{C}_{\hat{\mathbf{G}}}^{-1} \mathbf{F}\right)^{-1} \mathbf{F}^{\prime H} \\
& +\mathbf{F}\left(\frac{\partial}{\partial k^{*}}\left(\frac{\partial}{\partial k}\left(\mathbf{F}^{H} \mathbf{C}_{\hat{\mathbf{G}}}^{-1} \mathbf{F}\right)^{-1}\right)\right) \mathbf{F}^{H} \\
& \left.+\mathbf{F}\left(\frac{\partial}{\partial k}\left(\mathbf{F}^{H} \mathbf{C}_{\hat{\mathbf{G}}}^{-1} \mathbf{F}\right)^{-1}\right) \mathbf{F}^{\prime H}\right) \mathbf{C}_{\hat{\mathbf{G}}}^{-1} \hat{\mathbf{G}}
\end{aligned}
$$

where $\mathbf{F}^{\prime} \triangleq \partial \mathbf{F} / \partial k$. (21)-(22) can now be used to estimate $\hat{k}$ in (19) numerically.

A decent starting point for the numerical search is found by using the asymptotic approximation of the Hankel function given by [31]

$$
\mathcal{H}_{0}^{(1)}(x) \approx \sqrt{\frac{2}{\pi x}} e^{i(x-\pi / 4)}
$$

which leads to two (biased) problems linear in $k_{i}$ given by

$$
\begin{gathered}
\ln \left(\left|G^{\alpha}(r, \omega)\right|\right) \approx \ln \left(\left|A^{\alpha}\right|\right)+\ln \left(\left|\sqrt{\frac{2}{\pi k}}\right|\right)-\ln (\sqrt{r})-k_{i} r \\
\ln \left(\left|G^{\alpha}(r, \omega)\right|\right)+\ln (\sqrt{r}) \approx \ln \left(\left|A^{\alpha}\right|\right)+\ln \left(\left|\sqrt{\frac{2}{\pi k}}\right|\right)-k_{i} r \\
\ln \left(\sqrt{r}\left|G^{\alpha}(r, \omega)\right|\right) \approx \kappa-k_{i} r
\end{gathered}
$$

and similarly for $k_{r}$ through the phase of $G^{\alpha}$

$$
\begin{gathered}
\angle G^{\alpha}(r, \omega) \approx \angle A^{\alpha}-\angle \sqrt{k}-\pi / 4-k_{r} r \\
\angle G^{\alpha}(r, \omega)+\pi / 4 \approx \angle A^{\alpha}-\angle \sqrt{k}-k_{r} r \\
\angle G^{\alpha}(r, \omega)+\pi / 4 \approx \phi-k_{r} r
\end{gathered}
$$

which can be easily solved using linear regression.

The Fisher information matrix [29] is given by

$$
\mathcal{I}(\mathbf{A}, k)=\left[\begin{array}{cc}
\mathbf{F}^{H} \mathbf{C}_{\hat{\mathbf{G}}}^{-1} \mathbf{F} & \mathbf{F}^{H} \mathbf{C}_{\hat{\mathrm{G}}}^{-1} \mathbf{F}^{\prime} \mathbf{A} \\
\mathbf{A}^{H} \mathbf{F}^{\prime H} \mathbf{C}_{\hat{\mathbf{G}}}^{-1} \mathbf{F} & \mathbf{A}^{H} \mathbf{F}^{\prime H} \mathbf{C}_{\hat{\mathbf{G}}}^{-1} \mathbf{F}^{\prime} \mathbf{A}
\end{array}\right] .
$$

and finally, the Cramér-Rao lower bound and asymptotic covariance of $\hat{\mathbf{A}}$ and $\hat{k}$ are given by the inverse of the Fisher information matrix (23). 
2) Shear-Horizontal Waves: For SH modes, the derivation of the Maximum Likelihood estimator is based on (8). Since this is of the same form as (4), the resulting estimators are essentially the same as (19)-(23) but with

$$
\mathbf{F}\left(k^{y}\right)=\left[\begin{array}{c}
\mathcal{H}_{0}^{(1)}\left(-k^{y} r_{1}\right) \\
\vdots \\
\mathcal{H}_{0}^{(1)}\left(-k^{y} r_{M}\right)
\end{array}\right]
$$

and

$$
\mathbf{F}^{\prime}\left(k^{y}\right)=\left[\begin{array}{c}
r_{1} \mathcal{H}_{1}^{(1)}\left(-k^{y} r_{1}\right) \\
\vdots \\
r_{M} \mathcal{H}_{1}^{(1)}\left(-k^{y} r_{M}\right)
\end{array}\right]
$$

\section{Simulation}

\section{A. Simulation Setup}

In order to verify the derived estimation scheme and to assess its performance under ideal conditions, a series of Monte Carlo simulations are run. First, the number of measurement points $M$ is varied between $r_{1}=1 \mathrm{~m}$ and $r_{M}=10 \mathrm{~m}$ with $M=5$ and $M=10$. Furthermore, for each $M$, the number of repeated measurements $K$ is varied from $K=1$ up to $K=100$. Finally, for each $K$ - $M$-pair, a total of 1,000 simulations were run in order to verify the statistical properties. The input and output measurement noise variances were chosen as

$$
\sigma_{v}^{2}=\sigma_{w}^{2}=10 \times 10^{-3} .
$$

and the data was generated according to the model introduced in Section II. Finally, the true input signal $U$ was chosen to have magnitude 1 , that is, so that

$$
\mathbf{U}^{H} \mathbf{U}=1 \text {. }
$$

\section{B. Non-Parametric Frequency Response Function}

The simulation results for the estimator of the nonparametric FRF are shown in Fig. 4. The real and imaginary parts of the error $G-\hat{G}$ of the estimated FRF coefficient are shown in Fig. 4a and Fig. 4b, respectively, for two distances, $r_{1}=5 \mathrm{~m}$ and $r_{2}=10 \mathrm{~m}$. As it can be seen, the estimator converges to the true value for $K \approx 5$, that is, for only few experiments at the given distance.

Furthermore, the variance of $\hat{G}$ is shown and compared to the Cramér-Rao lower bound in Fig. 4c. First, note that the CRBs for the two distances are very close to each other and it is hence difficult to distinguish the two cases. Also, the variance converges rapidly to the theoretical lower limit and the estimator becomes efficient for low values of $K$. This is very beneficial since this reduces the time required for conducting experiments.

\section{Semi-Parametric Frequency Response Function}

Fig. 5 and Fig. 6 illustrate the simulation results for the estimators for $k$ and $A$, respectively. It can be seen from the mean estimation error that the estimators become unbiased for $K \approx 11$ for $M=5$ measurements and for $K \approx 8$ for $M=10$

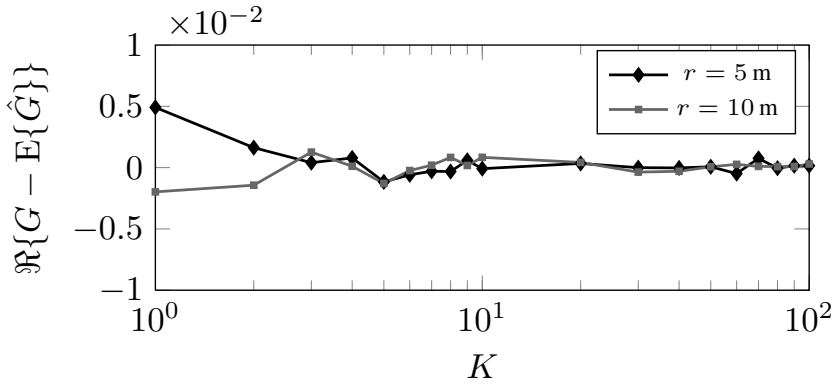

(a)

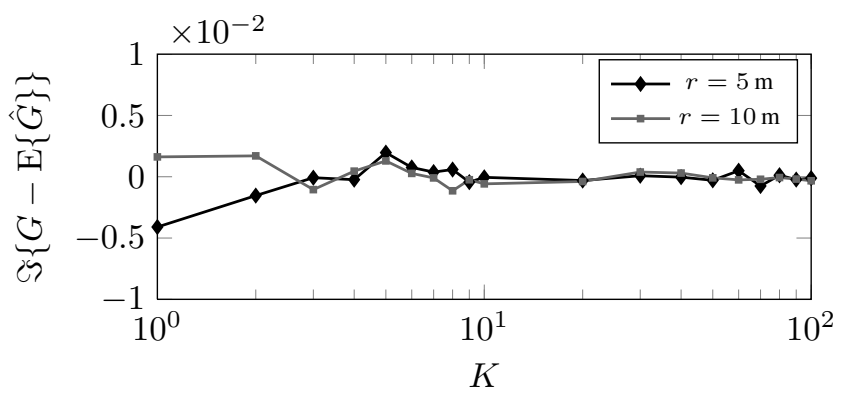

(b)

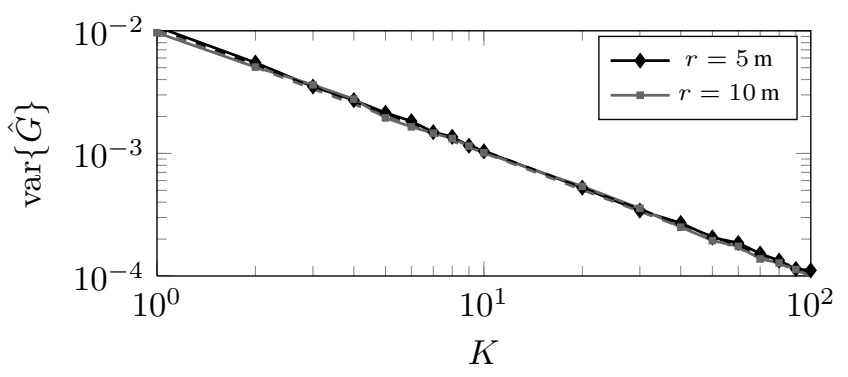

(c)

Fig. 4: Simulation results for $\hat{G}$ for different distances. (a) and (b) Mean estimation error $G-\hat{G}$ for the real and imaginary parts, respectively, and (c) Variance $\operatorname{var}\{\hat{G}\}$ (solid) and Cramér-Rao lower bound (dashed).

measurements. This is also confirmed by the comparison of the variance to the CRB which is attained around the same values for $K$ for either $M$.

From these simulations, it can be concluded that for the given noise levels, at least around $K \cdot M \approx 50$ measurements are required to obtain unbiased estimates that also attain the CRB.

\section{REsults}

Experiments using the setup outlined in Section III-A and Fig. 2 were conducted on two different occasions, once during a cold February day (ambient temperature approximately $-15^{\circ} \mathrm{C}$ ) and once on a spring day (ambient temperature approximately $15^{\circ} \mathrm{C}$ ) on a regular road in northern Sweden.OIn both occasions, the pavement was excited in $0.5 \mathrm{~m}$-intervals starting from $r_{1}=0.5 \mathrm{~m}$ up to $r_{M}=6 \mathrm{~m}$ yielding $M=12$ spatially separated measurement points. At each distance, the hammer was blown $K=10$ times. For both experiments, the sensors were mounted at exactly the same positions and also 


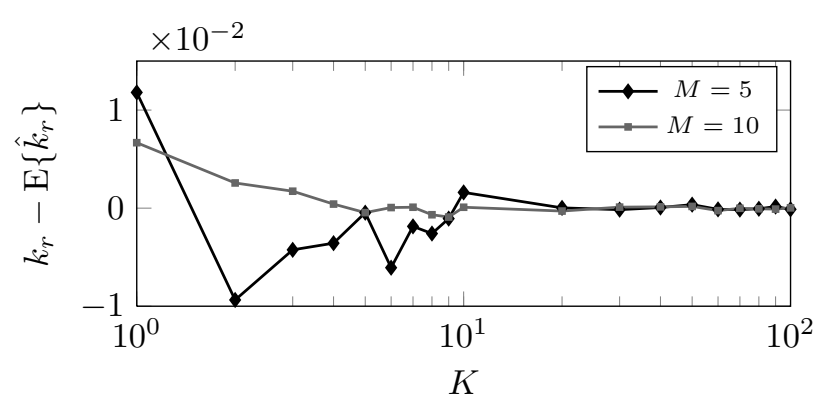

(a)

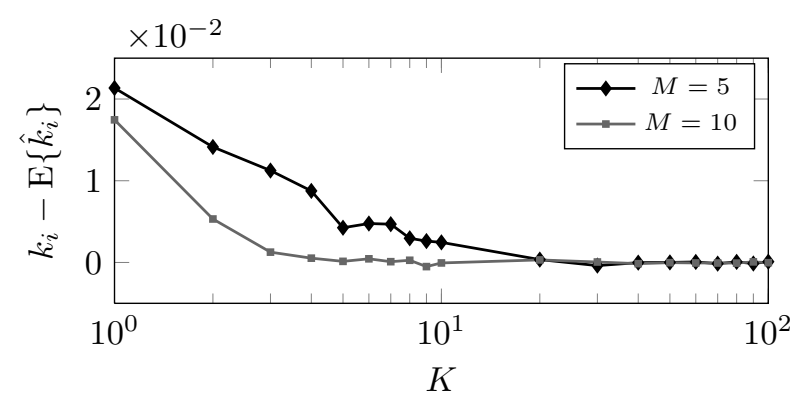

(b)

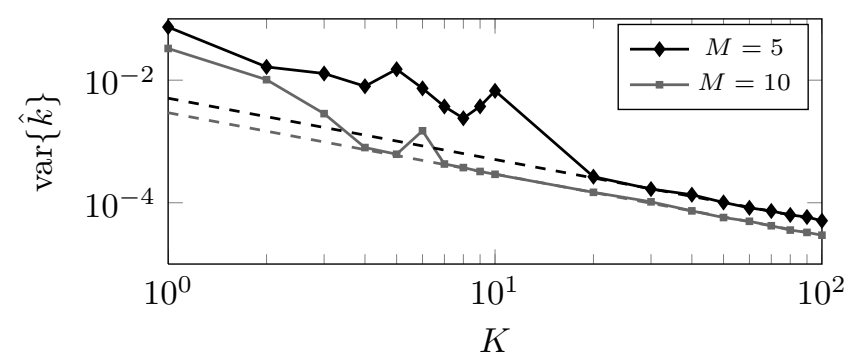

(c)

Fig. 5: Simulation results for $\hat{k}$ for varying $M$ and $K$. (a) and (b) Mean estimation error $k-\hat{k}$ for the real and imaginary parts, respectively, and (c) Variance $\operatorname{var}\{\hat{k}\}$ (solid) and Cramér-Rao lower bound (dashed).

the excitation points were chosen to be the same. The sensors were sampled at a sampling rate of $f_{s}=32.768 \mathrm{kHz}$.

\section{A. Input and Output Signal Analysis}

An example of the measured input and output spectra for the winter measurements is given in Fig. 7. In the depicted case, the distance between the source and sensor is $r=2 \mathrm{~m}$. It can be seen that the input energy is mostly concentrated below $800 \mathrm{~Hz}$ to $1 \mathrm{kHz}$. The response shown in Figure $7 \mathrm{~b}$ shows a relatively smooth trend following the excitation. However, a sharp peak is noted around $4 \mathrm{kHz}$ which indicates the existence of an eigenmode.

For the spring measurements shown in Fig. 8, one can see that the excitation is comparable to the excitation used in the winter experiments, with the main energy concentration below $1 \mathrm{kHz}$. The output, however, differs significantly from the output observed during cold conditions. While it is still quite smooth up to around $500 \mathrm{~Hz}$, there seems to be no or very little energy transmitted for frequencies above that point.

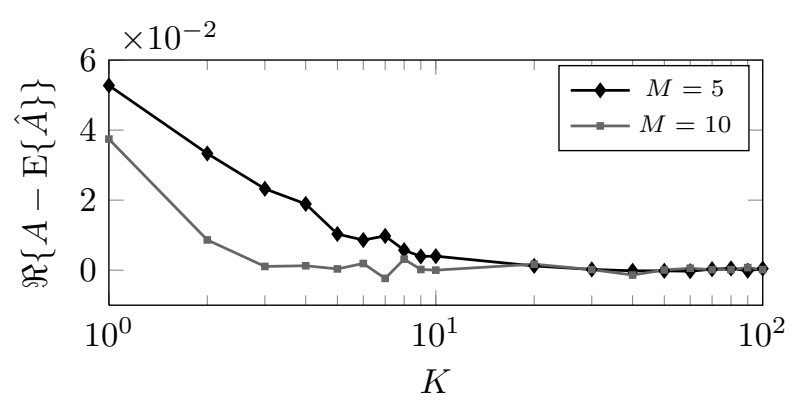

(a)

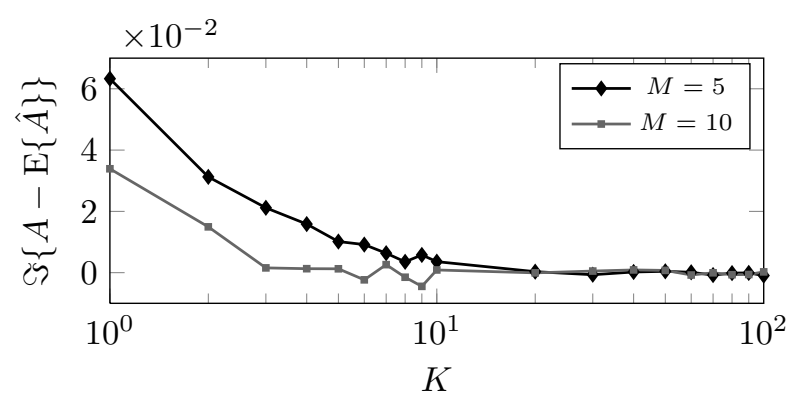

(b)

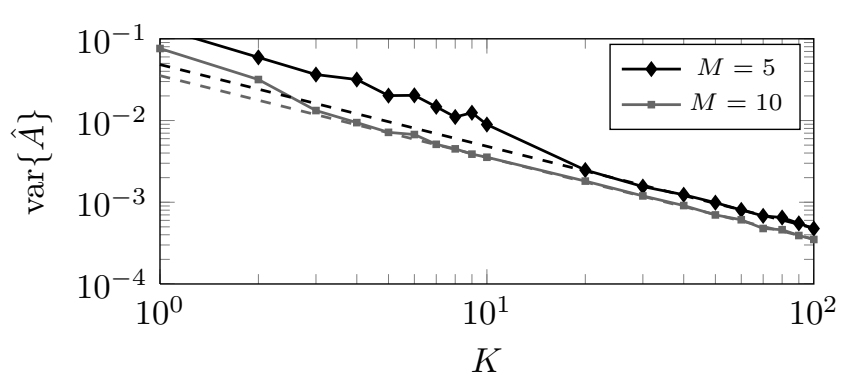

(c)

Fig. 6: Simulation results for $\hat{A}$ for varying $M$ and $K$. (a) and (b) Mean estimation error $A-\hat{A}$ for the real and imaginary parts, respectively, and (c) Variance $\operatorname{var}\{\hat{A}\}$ (solid) and Cramér-Rao lower bound (dashed).

Furthermore, the peak around $4 \mathrm{kHz}$ is not observed either.

\section{B. Non-Parametric Frequency Response Function}

Two examples for the non-parametric frequency response function estimated using (12) and as described in Section III-B are shown in Fig. 9 and Fig. 10 for both, winter and spring measurements.

For the winter measurements in Fig. 9 a smooth curve is obtained for both $r=2 \mathrm{~m}$ (Fig. 9a) and $r=4 \mathrm{~m}$ (Fig. 9b). Furthermore, the estimated FRF is significantly stronger than its variance and hence, good knowledge is obtained. Finally, it is difficult to see the spatial dampening from the shown plots. However, inspection of the peaks around $4 \mathrm{kHz}$ shows that the magnitude decreases about $5 \mathrm{~dB} / \mathrm{m}$ around these frequencies.

The spring measurements shown in Fig. 10 exhibit a quite different behavior. First, as it can be expected from the previous section's discussion, the estimated FRF is only smooth up to around $800 \mathrm{~Hz}$ and then becomes rather noisy. This is also reflected in the fact that starting from about 


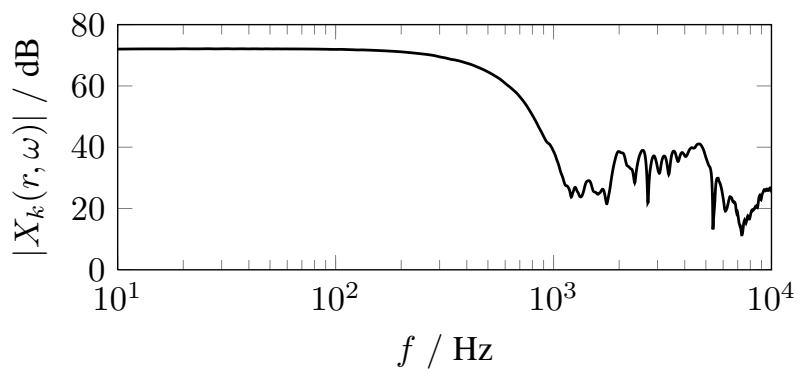

(a)

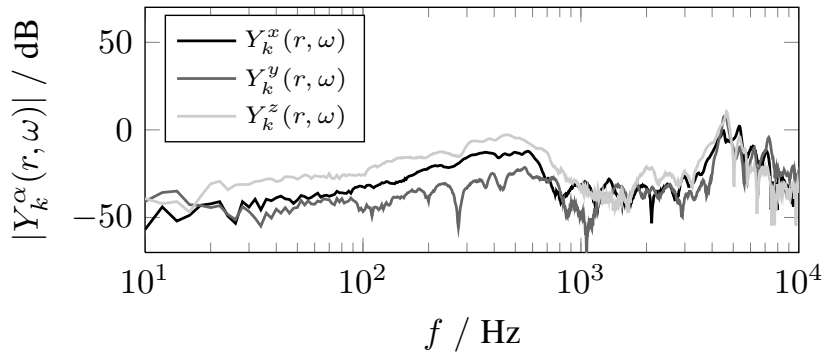

(b)

Fig. 7: Measured input (top) and output (bottom) spectra for $r=2 \mathrm{~m}$, winter measurements.

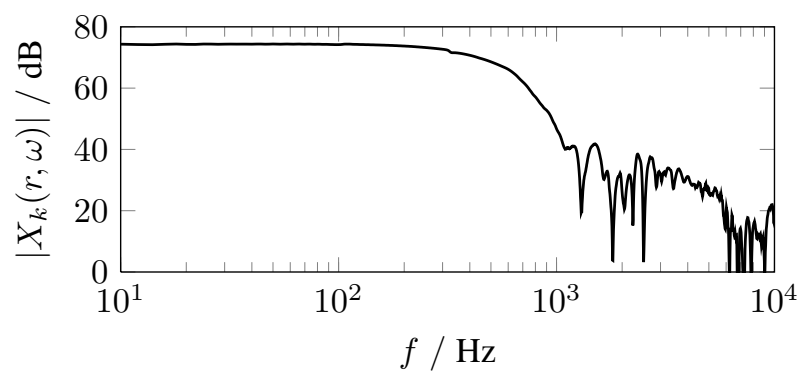

(a)

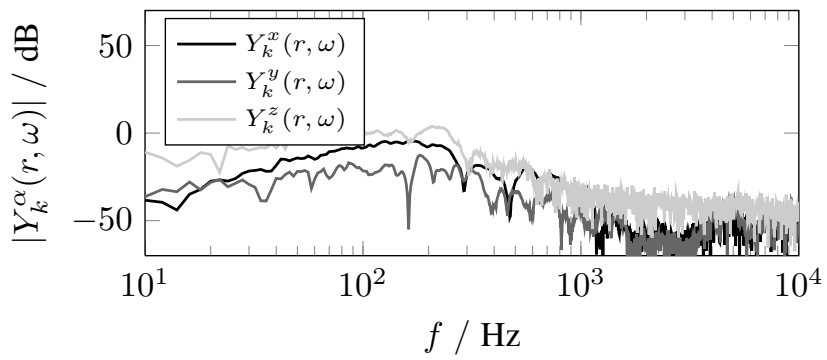

(b)

Fig. 8: Measured input (top) and output (bottom) spectra for $r=2 \mathrm{~m}$, spring measurements.

this frequency, there is essentially no difference between the estimated FRF and the noise, hence, only noise is measured. Second, comparing Fig. 10a and Fig. 10b, it is observed that with increasing distance, the frequency range that allows wave propagation is decreased.

Finally, one should note that the seasonal differences outlined above are not surprising since the temperature changes affect the properties of both, the asphalt concrete top plate

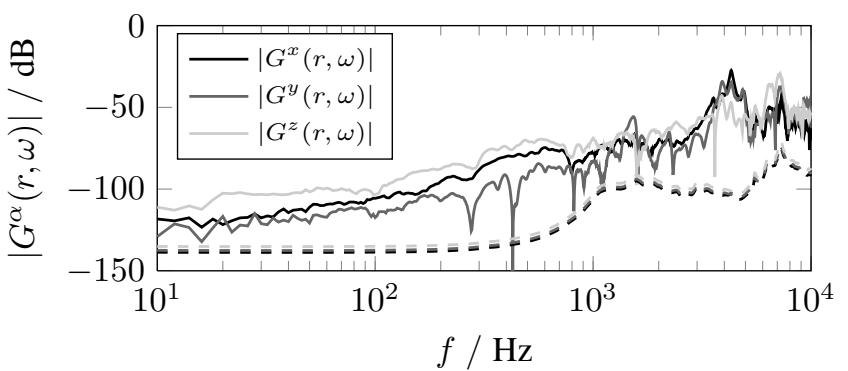

(a)

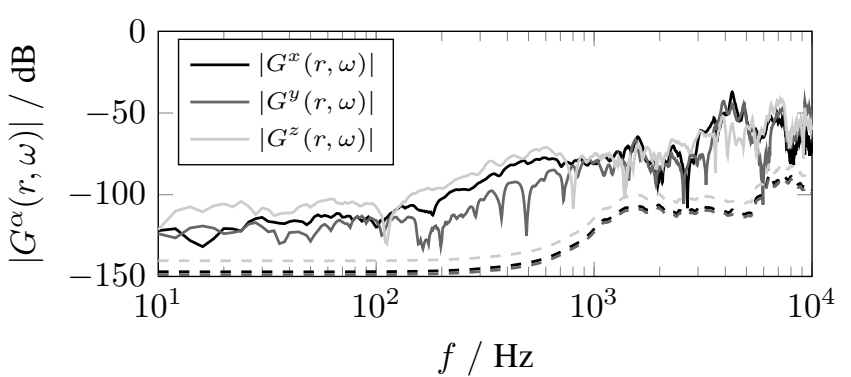

(b)

Fig. 9: Magnitude spectra of the estimated non-parametric frequency response function (solid) and their variances (dashed) for the winter measurements. (a) $r=2 \mathrm{~m}$, and (b) $r=4 \mathrm{~m}$.

as well as the subgrade layers. Colder temperatures clearly increase the stiffness of the material and hence, better wave transmission is obtained and vice-versa.

\section{Semi-Parametric Frequency Response Function}

Using the results from the previous section and the estimators (19)-(20) as described in Section III-C, the parameters of the semi-parametric FRF for the measurement data were estimated and are evaluated here. Note that in Fig. 11-Fig. 14 only frequencies where the SNR is larger than $25 \mathrm{~dB}$ and the coefficient of determination $R^{2}$ defined by

$$
R^{2}=1-\frac{\left(\hat{\mathbf{G}}-\overline{\boldsymbol{\mu}}_{\hat{\mathbf{G}}}\right)^{H}\left(\hat{\mathbf{G}}-\overline{\boldsymbol{\mu}}_{\hat{\mathbf{G}}}\right)}{(\hat{\mathbf{G}}-\mathbf{F}(\hat{k}) \hat{\mathbf{A}})^{H}(\hat{\mathbf{G}}-\mathbf{F}(\hat{k}) \hat{\mathbf{A}})}
$$

with $\bar{\mu}_{\mathrm{G}}$ the sample mean of $\hat{\mathrm{G}}$ is larger than 0.5 are shown. This ensures that the resulting graphs do not suffer from cluttering due to insignificant (noise-only) measurements since the only frequencies of interest are the ones where actual wave propagation takes place. In practice, this occurs around the eigenmodes and since these are relatively concentrated around certain frequencies, the frequencies in between will not be shown. Also note that changing either the threshold for the SNR or the $R^{2}$ value will affect the shown results.

1) Lamb Modes: Fig. 11 shows the estimated dispersion curves for Lamb modes, that is, the real part of the wavenumber $k=k_{r}-i k_{i}$ (Fig. 11a) and the phase velocity $c_{p}(\omega)=$ $\omega / k_{r}(\omega)$ (Fig. 11b) as functions of frequency, for the particle motion in the $x$ - and $z$-directions. It can be seen that different branches which can be attributed to different wave modes can be identified from the curve for the winter measurement 


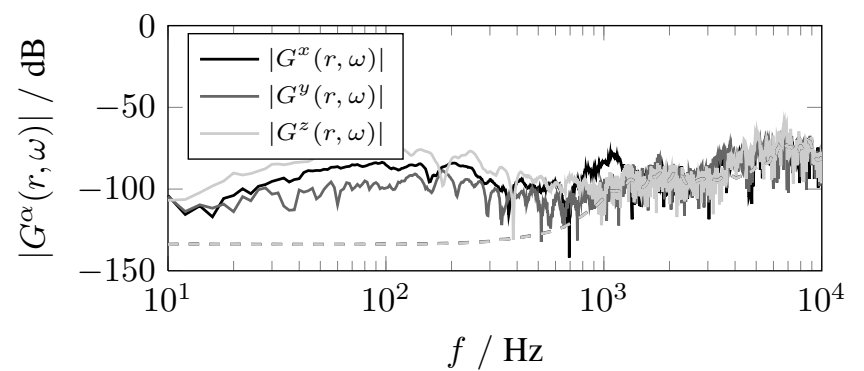

(a)

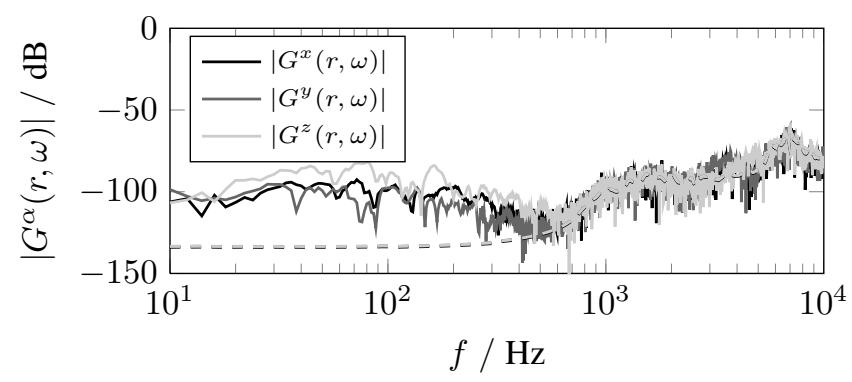

(b)

Fig. 10: Magnitude spectra of the estimated non-parametric frequency response function (solid) and their variances (dashed) for the spring measurements. (a) $r=2 \mathrm{~m}$, and (b) $r=4 \mathrm{~m}$.

(black). Furthermore, the different modes are observed for the whole frequency range considered and different modes are seen between $0 \mathrm{~Hz}$ and $1 \mathrm{kHz}, 2 \mathrm{kHz}$ and $5 \mathrm{kHz}$ (the jump around $4 \mathrm{kHz}$ can most likely be attributed to phase wrapping), and $8 \mathrm{kHz}$ and $10 \mathrm{kHz}$. For the spring measurements the previous observations are once again confirmed in the sense that fewer and basically only low-frequency modes are observed (gray). Further, phase wrapping is observed around $250 \mathrm{~Hz}$.

The parameters related to attenuation are shown in Fig. 12. However, interpretation of these is not as straight-forward as for the dispersion curves. For the modes identified earlier, it can be seen that the attenuation is particularly high (small $A^{x}$ and $A^{z}$ ) in the middle of the mode whereas it seems to be less towards the upper end of the mode, probably due to better decoupling from other modes. Also, there is very little information to be obtained for the spring measurements.

2) SH Modes: The dispersion curves for the second type of wave mode, shear horizontal waves, are shown in Fig. 13. It is obvious that these types of waves differ greatly from the Lamb modes discussed in the previous paragraph. For the winter measurements (black), the modes are much more scattered and the most predominant branch is found around $3 \mathrm{kHz}$, followed by what appears to be another major branch between $1 \mathrm{kHz}$ and $2 \mathrm{kHz}$ and one around $5 \mathrm{kHz}$, and finally, no propagation is observed above $5 \mathrm{kHz}$. Regarding the spring measurements (gray), similar observations are made but the branches are even more scattered and disjoint, except for one predominant branch around $3 \mathrm{kHz}$.

Considering the attenuation parameters shown in Fig. 14 similar observations are made. The coefficients are very scat-

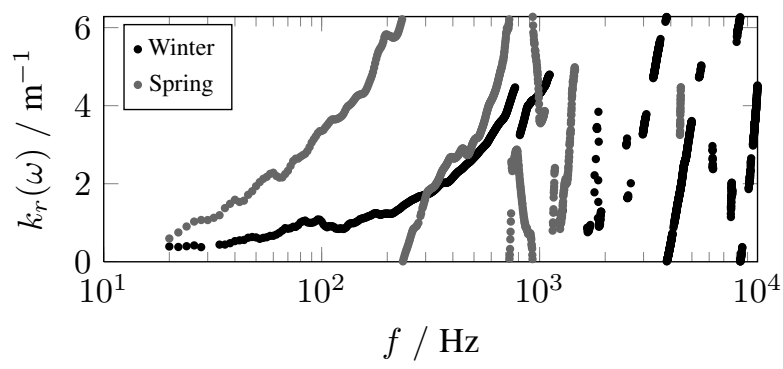

(a)

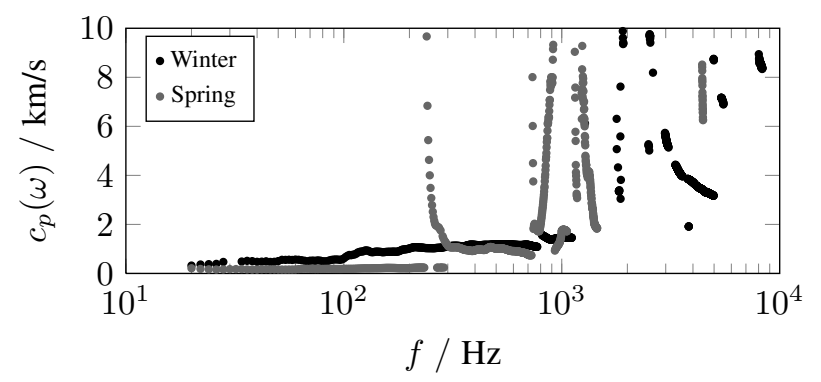

(b)

Fig. 11: Estimated dispersion curves for Lamb waves. (a) Real part of the wavenumber, and (b) phase velocity as functions of the frequency.

tered and little structure is observed.

\section{Discussion}

First, the results from applying the methods to the measurement data indicate that there is considerable variation in the material properties depending on the environmental conditions. This is reflected in the differences between the winter and spring measurements in both, the input-output signal analysis, the non-parametric FRF, and the semi-parametric FRF. Not surprisingly, the changing material properties affect all aspects of wave propagation: bandwidth of the propagated waves, propagation range (attenuation), and phase velocity. Thus, for a year-round application, it is important to carefully choose the frequency range in which the desired application should operate in order to guarantee a stable system.

Second, the proposed model gives reasonable results and is hence applicable. However, the model does not explicitly provide the mechanical parameters of the underlying system and can thus only be applied in cases where these parameters are unimportant. In order to estimate the mechanical parameters such as the elastic modulus, further processing would be required.

Third, the predominant wave mode encountered are Lamb waves which have their particle motion in-plane $(x)$ and outof-plane normal $(z)$ to the direction of wave propagation. This is no surprise since the excitation is also normal to the surface and thus, these are the preferred components to use for applications making use of waves in pavements.

\section{CONCLUSION}

It was shown how a statistical framework can be used for estimating the parameters of a model describing wave propa- 


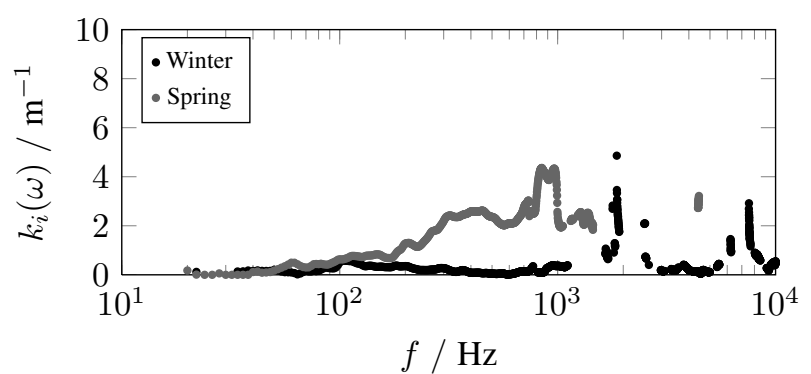

(a)

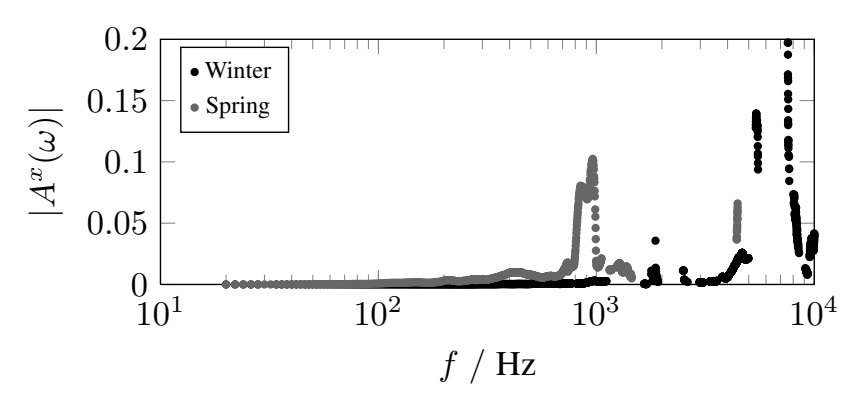

(b)

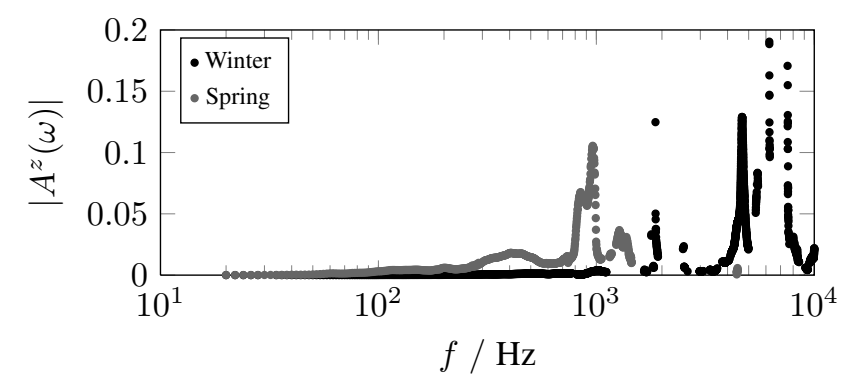

(c)

Fig. 12: Attenuation coefficients for Lamb modes, (a) $k_{i}$, (b) $A^{x}$, and (c) $A^{z}$.

gation in pavements from spatio-temporal experimental data. The model was derived from established theory and exhibits commonly encountered properties such as dispersive wave propagation and dampening. Then, estimators for the nonparametric frequency response function as well as the model shown earlier were derived. Evaluation of these estimators using experimental data obtained on two different occasions with varying environmental conditions showed that the method is feasible and revealed interesting details about the underlying structure.

An example for a future application of the proposed method could be the tuning of a road vibrations-based traffic monitoring system mentioned in the beginning. By conducting a couple of experiments as described here, the measurement system can then determine the initial material properties (attenuation and phase velocity) and use these in vehicle parameter estimation algorithms.

Finally, future work should consider a more complete parametric modeling, either using first principles or empirical modeling, of the frequency response function as well as modeling of the parameter variation. However, both tasks are

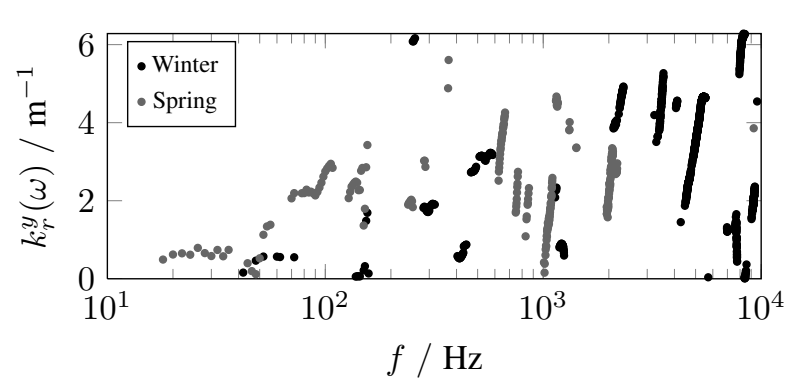

(a)

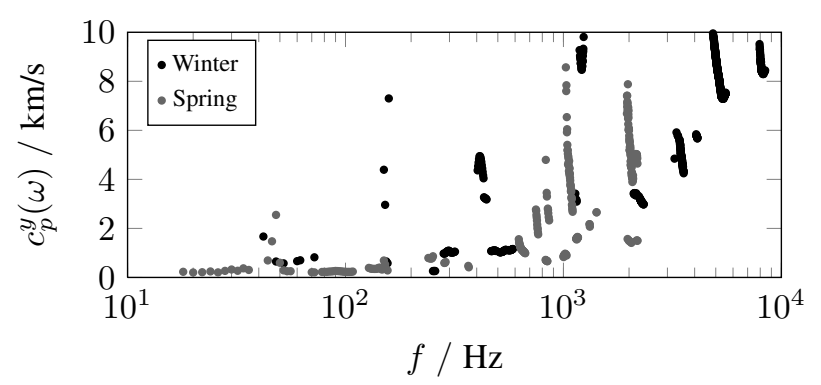

(b)

Fig. 13: Estimated dispersion curves for horizontal shear waves. (a) Real part of the wavenumber, and (b) phase velocity as functions of the frequency.

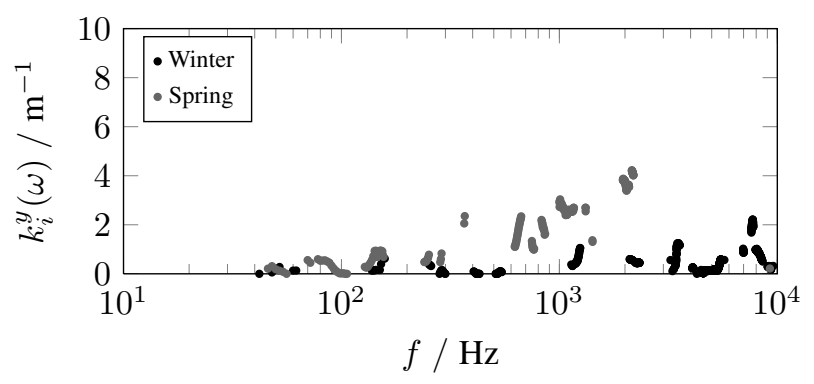

(a)

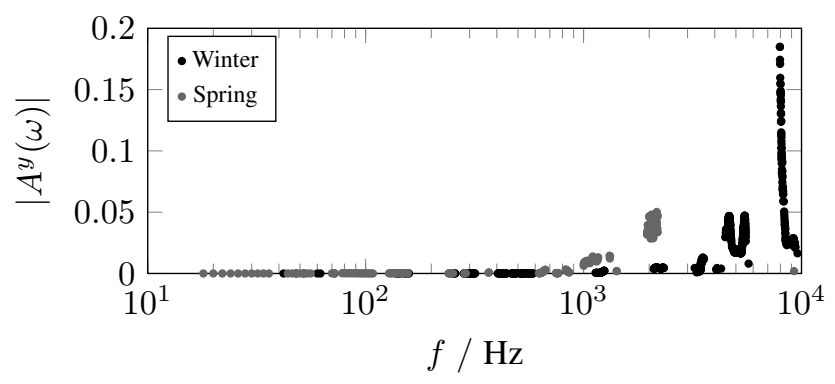

(b)

Fig. 14: Attenuation coefficients for horizontal shear wave modes, (a) $k_{i}^{y}$, and (b) $A^{y}$.

extremely challenging since the physics of the waveguide are non-trivial and the seasonal variation might be hard to measure with a reasonable effort.

\section{APPENDIX}

The joint PDF for the measured input $\mathbf{X}$ and output $\mathbf{Z}^{\alpha}$ is a multivariate complex Gaussian PDF given by (11). Thus, the 
maximum likelihood estimator for the unknown parameters $\boldsymbol{\theta}=\left[\begin{array}{ll}\mathbf{U}^{T} & G^{\alpha}\end{array}\right]^{T}$ is given by

$$
\hat{\boldsymbol{\theta}}=\underset{\boldsymbol{\theta}}{\operatorname{argmax}}-\left(\mathcal{X}-\boldsymbol{\mu}_{\mathcal{X}}(\boldsymbol{\theta})\right)^{H} \mathbf{C}_{\mathcal{X}}^{-1}\left(\mathcal{X}-\boldsymbol{\mu}_{\mathcal{X}}(\boldsymbol{\theta})\right)
$$

where

$$
\mathcal{X}=\left[\begin{array}{c}
\mathbf{X} \\
\mathbf{Z}^{\alpha}
\end{array}\right] \quad \text { and } \quad \mathbf{C}_{\mathcal{X}}=\left[\begin{array}{cc}
\mathbf{C}_{v v} & \mathbf{0} \\
\mathbf{0} & \mathbf{C}_{w w}
\end{array}\right]
$$

are introduced for simplicity. Also, the mean is given by

$$
\boldsymbol{\mu}_{\mathcal{X}}(\boldsymbol{\theta})=\left[\begin{array}{c}
\mathbf{U} \\
G^{\alpha} \mathbf{U}
\end{array}\right]=\left[\begin{array}{c}
\mathbf{I}_{K} \\
G^{\alpha} \mathbf{I}_{K}
\end{array}\right] \mathbf{U}=\mathbf{H}\left(G^{\alpha}\right) \mathbf{U}
$$

which is linear in $\mathbf{U}$ and hence, the problem is separable. The MLE for U given $G^{\alpha}$ is thus [29]

$$
\hat{\mathbf{U}}=\left(\mathbf{H}^{H}\left(G^{\alpha}\right) \mathbf{C}_{\mathcal{X}}^{-1} \mathbf{H}\left(G^{\alpha}\right)\right)^{-1} \mathbf{H}^{H}\left(G^{\alpha}\right) \mathbf{C}_{\mathcal{X}}^{-1} \mathcal{X}
$$

Substituting (27) in (24) yields the cost function

$$
J\left(G^{\alpha}\right)=\mathcal{X}^{H} \mathbf{C}_{\mathcal{X}}^{-1} \mathbf{H}\left(\mathbf{H}^{H} \mathbf{C}_{\mathcal{X}}^{-1} \mathbf{H}\right)^{-1} \mathbf{H}^{H} \mathbf{C}_{\mathcal{X}}^{-1} \mathcal{X}
$$

for $G^{\alpha}$ where $\mathbf{H} \triangleq \mathbf{H}\left(G^{\alpha}\right)$. The extrema of (28) are found by deriving with respect to the complex conjugate of $G^{\alpha}$ as defined in [30] and equating it to zero.

First, note that

$$
\frac{\partial}{\partial\left(G^{\alpha}\right)^{*}} \mathbf{H}=0
$$

since $\mathbf{H}$ does not depend on $\left(G^{\alpha}\right)^{*}$ and

$$
\frac{\partial}{\partial\left(G^{\alpha}\right)^{*}} \mathbf{H}^{H}=\left(\frac{\partial}{\partial G^{\alpha}} \mathbf{H}\right)^{H}=\left[\begin{array}{ll}
\mathbf{0} & \mathbf{I}_{K}
\end{array}\right] .
$$

Finally, we denote the derivative of $\mathbf{H}$ with respect to $G^{\alpha}$ as $\mathbf{H}^{\prime}$, that is,

$$
\mathbf{H}^{\prime} \triangleq \frac{\partial}{\partial G^{\alpha}} \mathbf{H}
$$

$$
\begin{aligned}
& \text { Using (29) then yields } \\
& \begin{array}{l}
\frac{\partial J\left(G^{\alpha}\right)}{\partial\left(G^{\alpha}\right)^{*}}=\frac{\partial}{\partial\left(G^{\alpha}\right)^{*}} \mathcal{X}^{H} \mathbf{C}_{\mathcal{X}}^{-1} \mathbf{H}\left(\mathbf{H}^{H} \mathbf{C}_{\mathcal{X}}^{-1} \mathbf{H}\right)^{-1} \mathbf{H}^{H} \mathbf{C}_{\mathcal{X}}^{-1} \mathcal{X} \\
=\mathcal{X}^{H} \mathbf{C}_{\mathcal{X}}^{-1} \mathbf{H}\left(\frac{\partial}{\partial\left(G^{\alpha}\right)^{*}}\left(\mathbf{H}^{H} \mathbf{C}_{\mathcal{X}}^{-1} \mathbf{H}\right)^{-1} \mathbf{H}^{H}\right) \mathbf{C}_{\mathcal{X}}^{-1} \mathcal{X}
\end{array}
\end{aligned}
$$

for the derivative of the cost function. Furthermore, applying the product rule gives

$$
\begin{gathered}
\frac{\partial}{\partial\left(G^{\alpha}\right)^{*}}\left(\mathbf{H}^{H} \mathbf{C}_{\mathcal{X}}^{-1} \mathbf{H}\right)^{-1} \mathbf{H}^{H}=\left(\frac{\partial}{\partial\left(G^{\alpha}\right)^{*}}\left(\mathbf{H}^{H} \mathbf{C}_{\mathcal{X}}^{-1} \mathbf{H}\right)^{-1}\right) \\
\cdot \mathbf{H}^{H}+\left(\mathbf{H}^{H} \mathbf{C}_{\mathcal{X}}^{-1} \mathbf{H}\right)^{-1} \frac{\partial}{\partial\left(G^{\alpha}\right)^{*}} \mathbf{H}^{H}
\end{gathered}
$$

and

$$
\begin{aligned}
\frac{\partial}{\partial\left(G^{\alpha}\right)^{*}}\left(\mathbf{H}^{H} \mathbf{C}_{\mathcal{X}}^{-1} \mathbf{H}\right)^{-1}=-\left(\mathbf{H}^{H} \mathbf{C}_{\mathcal{X}}^{-1} \mathbf{H}\right)^{-1} \\
\cdot\left(\left(\frac{\partial}{\partial\left(G^{\alpha}\right)^{*}} \mathbf{H}^{H}\right) \mathbf{C}_{\mathcal{X}}^{-1} \mathbf{H}\right)\left(\mathbf{H}^{H} \mathbf{C}_{\mathcal{X}}^{-1} \mathbf{H}\right)^{-1}
\end{aligned}
$$

Finally, the derivative becomes

$$
\begin{array}{r}
\frac{\partial J\left(G^{\alpha}\right)}{\partial\left(G^{\alpha}\right)^{*}}=\mathcal{X}^{H} \mathbf{C}_{\mathcal{X}}^{-1} \mathbf{H}\left(\mathbf{H}^{H} \mathbf{C}_{\mathcal{X}}^{-1} \mathbf{H}\right)^{-1} \mathbf{H}^{\prime H} \mathbf{C}_{\mathcal{X}}^{-1} \mathcal{X} \\
-\mathcal{X}^{H} \mathbf{C}_{\mathcal{X}}^{-1} \mathbf{H}\left(\mathbf{H}^{H} \mathbf{C}_{\mathcal{X}}^{-1} \mathbf{H}\right)^{-1}\left(\mathbf{H}^{\prime H} \mathbf{C}_{\mathcal{X}}^{-1} \mathbf{H}\right) \\
\cdot\left(\mathbf{H}^{H} \mathbf{C}_{\mathcal{X}}^{-1} \mathbf{H}\right)^{-1} \mathbf{H}^{H} \mathbf{C}_{\mathcal{X}}^{-1} \mathcal{X}
\end{array}
$$

Substituting the values for $\mathcal{X}, \mathbf{C}_{\mathcal{X}}, \mathbf{H}$, and $\mathbf{H}^{\prime}$ gives

$$
\begin{aligned}
\mathcal{X}^{H} \mathbf{C}_{\mathcal{X}}^{-1} \mathbf{H} & =\left[\begin{array}{ll}
\mathbf{X}^{H} & \left(\mathbf{Z}^{\alpha}\right)^{H}
\end{array}\right]\left[\begin{array}{cc}
\mathbf{C}_{v v}^{-1} & \mathbf{0} \\
\mathbf{0} & \mathbf{C}_{w w}^{-1}
\end{array}\right]\left[\begin{array}{c}
\mathbf{I}_{K} \\
G^{\alpha} \mathbf{I}_{K}
\end{array}\right] \\
& =\frac{\mathbf{X}^{H}}{\sigma_{v}^{2}}+\frac{G^{\alpha}\left(\mathbf{Z}^{\alpha}\right)^{H}}{\sigma_{w}^{2}} \\
\mathbf{H}^{H} \mathbf{C}_{\mathcal{X}}^{-1} \mathbf{H} & =\left[\begin{array}{ll}
\mathbf{I}_{K}^{H} & \left(G^{\alpha} \mathbf{I}_{K}\right)^{H}
\end{array}\right]\left[\begin{array}{cc}
\mathbf{C}_{v v}^{-1} & \mathbf{0} \\
\mathbf{0} & \mathbf{C}_{w w}^{-1}
\end{array}\right]\left[\begin{array}{c}
\mathbf{I}_{K} \\
G^{\alpha} \mathbf{I}_{K}
\end{array}\right] \\
& =\frac{1}{\sigma_{v}^{2}}+\frac{G^{\alpha}\left(G^{\alpha}\right)^{*}}{\sigma_{w}^{2}} \\
\mathbf{H}^{\prime H} \mathbf{C}_{\mathcal{X}}^{-1} \mathcal{X} & =\left[\begin{array}{ll}
\mathbf{0} & \mathbf{I}_{K}
\end{array}\right]\left[\begin{array}{cc}
\mathbf{C}_{v v}^{-1} & \mathbf{0} \\
\mathbf{0} & \mathbf{C}_{w w}^{-1}
\end{array}\right]\left[\begin{array}{c}
\mathbf{X} \\
\mathbf{Z}^{\alpha}
\end{array}\right]=\frac{\mathbf{Z}^{\alpha}}{\sigma_{w}^{2}} \\
\mathbf{H}^{\prime H} \mathbf{C}_{\mathcal{X}}^{-1} \mathbf{H} & =\left[\begin{array}{ll}
\mathbf{0} & \mathbf{I}_{K}
\end{array}\right]\left[\begin{array}{cc}
\mathbf{C}_{v v}^{-1} & \mathbf{0} \\
\mathbf{0} & \mathbf{C}_{w w}^{-1}
\end{array}\right]\left[\begin{array}{c}
\mathbf{I}_{K} \\
G^{\alpha} \mathbf{I}_{K}
\end{array}\right]=\frac{G^{\alpha}}{\sigma_{w}}
\end{aligned}
$$

and

$$
\mathbf{H}^{H} \mathbf{C}_{\mathcal{X}}^{-1} \mathcal{X}=\left(\mathcal{X}^{H} \mathbf{C}_{\mathcal{X}}^{-1} \mathbf{H}\right)^{H}=\frac{\mathbf{X}}{\sigma_{v}^{2}}+\frac{\left(G^{\alpha}\right)^{*} \mathbf{Z}^{\alpha}}{\sigma_{w}^{2}}
$$

which finally gives

$$
\begin{aligned}
& \frac{\partial J\left(G^{\alpha}\right)}{\partial\left(G^{\alpha}\right)^{*}}=\left(\frac{1}{\sigma_{v}^{2}}+\frac{G^{\alpha}\left(G^{\alpha}\right)^{*}}{\sigma_{w}^{2}}\right)^{-1} \\
& \cdot\left(\frac{\mathbf{X}^{H}}{\sigma_{v}^{2}}+\frac{G^{\alpha}\left(\mathbf{Z}^{\alpha}\right)^{H}}{\sigma_{w}^{2}}\right) \frac{\mathbf{Z}^{\alpha}}{\sigma_{w}^{2}}-\left(\frac{1}{\sigma_{v}^{2}}+\frac{G^{\alpha}\left(G^{\alpha}\right)^{*}}{\sigma_{w}^{2}}\right)^{-2} \\
& \cdot \frac{G^{\alpha}}{\sigma_{w}}\left(\frac{\mathbf{X}^{H}}{\sigma_{v}^{2}}+\frac{G^{\alpha}\left(\mathbf{Z}^{\alpha}\right)^{H}}{\sigma_{w}^{2}}\right)\left(\frac{\mathbf{X}}{\sigma_{v}^{2}}+\frac{\left(G^{\alpha}\right)^{*} \mathbf{Z}^{\alpha}}{\sigma_{w}^{2}}\right)
\end{aligned}
$$

Setting (31) equal to zero, expanding and simplifying finally yields

$0=\left(G^{\alpha}\right)^{2} \frac{\left(\mathbf{Z}^{\alpha}\right)^{H} \mathbf{X}}{\sigma_{w}^{2}}+G^{\alpha}\left(\frac{\mathbf{X}^{H} \mathbf{X}}{\sigma_{v}^{2}}-\frac{\left(\mathbf{Z}^{\alpha}\right)^{H} \mathbf{Z}^{\alpha}}{\sigma_{w}^{2}}\right)-\frac{\mathbf{X}^{H} \mathbf{Z}^{\alpha}}{\sigma_{v}^{2}}$

which is quadratic in $G^{\alpha}$ and hence, $J\left(G^{\alpha}\right)$ has two finite extrema given by

$$
\hat{G}^{\alpha}=\frac{-b \pm \sqrt{b^{2}-4 a c}}{2 a}
$$

where

$$
\begin{aligned}
& a=\frac{\left(\mathbf{Z}^{\alpha}\right)^{H} \mathbf{X}}{\sigma_{w}^{2}} \\
& b=\frac{\mathbf{X}^{H} \mathbf{X}}{\sigma_{v}^{2}}-\frac{\left(\mathbf{Z}^{\alpha}\right)^{H} \mathbf{Z}^{\alpha}}{\sigma_{w}^{2}} \\
& c=-\frac{\mathbf{X}^{H} \mathbf{Z}^{\alpha}}{\sigma_{v}^{2}}
\end{aligned}
$$

It is not straight forward to see which of the two solutions is the maximum and minimum, respectively. However, it is not expensive to calculate both, evaluate the cost (24), and then 
select the maximum from the two possibilities. Also, note that $b$ is a real number and $-4 a c$ is a real, positive number.

The Fisher information matrix for $\left\{\mathbf{U}, G^{\alpha}\right\}$ can be partitioned according to

$$
\mathcal{I}\left(\mathbf{U}, G^{\alpha}\right)=\left[\begin{array}{ll}
\mathcal{I}_{\mathbf{U U}} & \mathcal{I}_{\mathbf{U} G^{\alpha}} \\
\mathcal{I}_{G^{\alpha} \mathbf{U}} & \mathcal{I}_{G^{\alpha} G^{\alpha}}
\end{array}\right]
$$

where the submatrices are given by

$$
\begin{aligned}
& \mathcal{I}_{\mathbf{U U}}=\mathrm{E}\left\{\frac{\partial \ln (p(\mathbf{Z} ; \boldsymbol{\theta}))}{\partial \mathbf{U}^{*}}\left(\frac{\partial \ln (p(\mathbf{Z} ; \boldsymbol{\theta}))}{\partial \mathbf{U}^{*}}\right)^{H}\right\} \\
& \mathcal{I}_{\mathbf{U} G^{\alpha}}=\mathrm{E}\left\{\frac{\partial \ln (p(\mathbf{Z} ; \boldsymbol{\theta}))}{\partial \mathbf{U}^{*}}\left(\frac{\partial \ln (p(\mathbf{Z} ; \boldsymbol{\theta}))}{\partial\left(G^{\alpha}\right)^{*}}\right)^{H}\right\} \\
& \mathcal{I}_{G^{\alpha} \mathbf{U}}=\mathcal{I}_{\mathbf{U} G^{\alpha}}^{H} \\
& \mathcal{I}_{G^{\alpha} G^{\alpha}}=\mathrm{E}\left\{\frac{\partial \ln (p(\mathbf{Z} ; \boldsymbol{\theta}))}{\partial\left(G^{\alpha}\right)^{*}}\left(\frac{\partial \ln (p(\mathbf{Z} ; \boldsymbol{\theta}))}{\partial\left(G^{\alpha}\right)^{*}}\right)^{H}\right\}
\end{aligned}
$$

which finally gives

$$
\mathcal{I}\left(\mathbf{U}, G^{\alpha}\right)=\left[\begin{array}{cc}
\mathbf{H}^{H} \mathbf{C}_{\mathcal{X}}^{-1} \mathbf{H} & \mathbf{H}^{H} \mathbf{C}_{\mathcal{X}}^{-1} \mathbf{H}^{\prime} \mathbf{U} \\
\mathbf{U}^{H} \mathbf{H}^{\prime H} \mathbf{C}_{\mathcal{X}}^{-1} \mathbf{H} & \mathbf{U}^{H} \mathbf{H}^{\prime H} \mathbf{C}_{\mathcal{X}}^{-1} \mathbf{H}^{\prime} \mathbf{U}
\end{array}\right]
$$

and the covariance is thus bounded by the Cramér-Rao lower bound (CRLB) as the inverse of (33) [29]. Note that since the true $\mathbf{U}$ and $G^{\alpha}$ are unknown, the asymptotic covariance can be estimated using the estimated $\hat{\mathbf{U}}$ and $\hat{G}^{\alpha}$ instead.

\section{REFERENCES}

[1] R. Hostettler, M. Lundberg Nordenvaad, and W. Birk, "The pavement as a waveguide: Modeling, system identification, and parameter estimation," Instrumentation and Measurement, IEEE Transactions on, vol. 63, no. 8, pp. 2052-2063, 2014.

[2] A. B. Goktepe, E. Agar, and A. H. Lav, "Advances in backcalculating the mechanical properties of flexible pavements," Advances in Engineering Software, vol. 37, no. 7, pp. 421-431, 2006.

[3] A. Goel and A. Das, "A brief review on different surface wave methods and their applicability for non-destructive evaluation of pavements," in 2006 Highway Geophysics-NDE Conference, December 2006, pp. 337 350 .

[4] B. McCall and W. C. Vodrazka, States' Successful Practices Weigh-inMotion Handbook. Federal Highway Administration, 1997.

[5] R. Hostettler, W. Birk, and M. Lundberg Nordenvaad, "Feasibility of road vibrations-based vehicle property sensing," Intelligent Transport Systems, IET, vol. 4, no. 4, pp. 356-364, December 2010.

[6] R. Bajwa, R. Rajagopal, P. Varaiya, and R. Kavaler, "In-pavement wireless sensor network for vehicle classification," in Information Processing in Sensor Networks (IPSN), 2011 10th International Conference on, Chicago, IL, USA, April 2011, pp. 85-96.

[7] A. I. Claessen, C. P. Valkering, and R. Ditmarsch, "Pavement evaluation with the falling weight deflectometer," in Association of Asphalt Paving Technologists Proc, vol. 45, 1976.

[8] M. S. Hoffman and M. R. Thompson, "Comparative study of selected nondestructive testing devices," Transportation Research Record, no. 852 , pp. 32-41, 1982.

[9] J. S. Heisey, K. H. Stokoe, and A. H. Meyer, "Moduli of pavement systems from spectral analysis of surface waves," Transportation Research Record, no. 852, pp. 22-31, 1982.

[10] C. B. Park, R. D. Miller, and J. Xia, "Multichannel analysis of surface waves," Geophysics, vol. 64, no. 3, pp. 800-808, 1999.

[11] N. Ryden, C. B. Park, P. Ulriksen, and R. D. Miller, "Multimodal approach to seismic pavement testing," Journal of Geotechnical and Geoenvironmental Engineering, vol. 130, no. 6, pp. 636-645, 2004.

[12] S. D. Tayabji and E. O. E. Lukanen, Nondestructive Testing of Pavements and Backcalculations of Moduli, 2000, vol. 3.

[13] L. H. Irwin, "Backcalculation: An overview and perspective," in Pavement Evaluation Conference, Roanoke, VA, USA, 2002.
[14] L. Shuo, T. F. Fwa, and K. H. Tan, "Closed-form back-calculation of rigid-pavement parameters," Journal of Transportation Engineering, vol. 122, no. 1 , pp. 5-11, 1996.

[15] J. Uzan, "Dynamic linear back calculation of pavement material parameters," Journal of Transportation Engineering, vol. 120, no. 1, pp. 109-126, 1994.

[16] T. F. Fwa, C. Y. Tan, and W. T. Chan, "Backcalculation analysis of pavement-layer moduli using genetic algorithms," Transportation Research Record: Journal of the Transportation Research Board, vol. 1570, no. 1, pp. 134-142, 1997.

[17] O. Hunaidi, "Evolution-based genetic algorithms for analysis of nondestructive surface wave tests on pavements," NDT \& E International, vol. 31, no. 4, pp. 273-280, 1998.

[18] L. Khazanovich and J. Roesler, "Diploback: Neural-network-based backcalculation program for composite pavements," Transportation Research Record: Journal of the Transportation Research Board, vol. 1570, no. 1, pp. 143-150, 1997.

[19] N. Rakesh, A. K. Jain, M. A. Reddy, and K. S. Reddy, "Artificial neural networks - genetic algorithm based model for backcalculation of pavement layer moduli," International Journal of Pavement Engineering, vol. 7, no. 3, pp. 221-230, 2006.

[20] H. Lamb, "On waves in an elastic plate," Proceedings of the Royal Society of London. Series A, vol. 93, no. 648, pp. 114-128, March 1917.

[21] L. P. Solie and B. A. Auld, "Elastic waves in free anisotropic plates," The Journal of the Acoustical Society of America, vol. 54, no. 1, pp. 50-65, 1973.

[22] J. F. Doyle, Wave Propagation in Structures - Spectral Analysis Using Fast Discrete Fourier Transforms. New York, NY, USA: Springer, 1997.

[23] J. L. Rose, Ultrasonic Waves in Solid Media. Cambridge, UK: Cambridge University Press, 1999.

[24] L. Ljung, System Identification: Theory for the User. Upper Saddle River, NJ, USA: Prentice Hall, 1997.

[25] R. Pintelon and J. Schoukens, System Identification: A Frequency Domain Approach. Piscataway, NJ, USA: Wiley-IEEE Press, 2001.

[26] _ - "Measurement and modelling of linear systems in the presence of non-linear distortions," Mechanical Systems and Signal Processing, vol. 16, no. 5, pp. 785-801, 2002.

[27] S. Nazarian, K. H. Stokoe, and R. W. Hudson, "Use of spectral analysis of surface waves method for determination of moduli and thicknesses of pavement systems," Transportation Research Record, no. 930, pp. 38-45, 1983.

[28] N. Ryden, P. Ulriksen, C. B. Park, R. D. Miller, J. Xia, and J. Ivanov, "High frequency MASW for non-destructive testing of pavements - accelerometer approach," in Symposium on the Application of Geophysics to Engineering and Environmental Problems (SAGEEP 2002), 2001.

[29] S. M. Kay, Fundamentals of Statistical Signal Processing: Estimation Theory. Upper Saddle River, NJ, USA: Prentice Hall, 1993.

[30] A. Van Den Bos, "Complex gradient and Hessian," Vision, Image and Signal Processing, IEE Proceedings on, vol. 141, no. 6, pp. 380-383, 1994.

[31] M. Abramowitz and I. A. Stegun, Handbook of Mathematical Functions. Washington, DC, USA: National Institute of Standards and Technology, 1964.

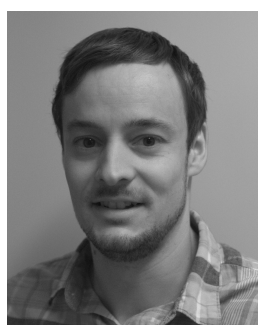

Roland Hostettler (S'10) received the Dipl. Ing. degree in electrical and communication engineering from Bern University of Applied Sciences, Switzerland in 2007 and the M.Sc. deegree in electrical engineering from Luleå University of Technology, Sweden in 2009

$\mathrm{He}$ is currently working toward the Ph.D. degree in automatic control at Luleå University of Technology, Sweden. His research interests include parameter and state estimation, and system identification. 


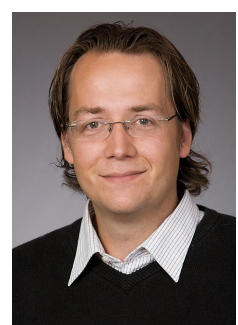

Magnus Lundberg Nordenvaad (M'07) was born in Luleå, Sweden, in 1973. He received the M.Sc degree in computer science and engineering from Luleå University of Technology (LTU), Luleå, Sweden, in 1998 and the Ph.D. degree in signal processing from the School of Electrical and Computer Engineering, Chalmers University of Technology, Gothenburg, Sweden, in 2003.

$\mathrm{He}$ has held visiting positions at Purdue University, Colorado State University, and the University of Florida. Currently, he is a Senior Lecturer with the Department of Information Technology, Uppsala University, Uppsala, Sweden, and holds a senior research position at the Swedish Defence Research Agency. His research interests lie in statistical signal processing and how it applies to digital communications, radar, sonar, process diagnostics, and land-mine detection.

Dr. Nordenvaad received the 1998 MD110 User-Group Award for the Best Master's Thesis in the telecommunications area in Sweden that year and a post-doctoral scholarship award from the Swedish Research Council, among other honors.

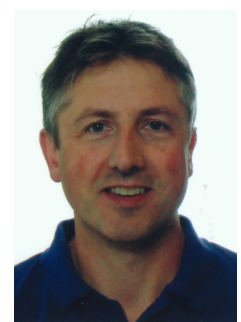

Wolfgang Birk (M'97) is currently Associate Professor at the Control Engineering Group at Luleå University of Technology. He holds a M.Sc. degree in Electrical Engineering from University of Saarland, Germany (1997) and a Ph.D. degree in Automatic Control from Luleå University of Technology (2002). Birk has a background in the development of Active Safety system of passenger cars, relating to collision avoidance, collision mitigation, and driver state estimation and warning. For his work relating to the development of Volvo Cars Driver Alert, he has received the Henry Ford Technology Award (2007). Birk is co-founder of several spin-off companies from research projects and is actively involved in research and development activities relating to intelligent transport systems, control of complex interconnected systems and process control applications. 